\title{
The unique mode of action of a divergent member of the ABA-receptor protein family in ABA and stress signaling
}

Yang Zhao ${ }^{1,2, *}$, Zhulong Chan ${ }^{1,3, *}$, Lu Xing ${ }^{2,4}$, Xiaodong Liu ${ }^{1}$, Yueh-Ju Hou ${ }^{2}$, Viswanathan Chinnusamy ${ }^{2,5}$, Pengcheng Wang ${ }^{2}$, Chengguo Duan ${ }^{2}$, Jian-Kang Zhu ${ }^{1,2}$

${ }^{I}$ Shanghai Center for Plant Stress Biology and Shanghai Institute of Plant Physiology and Ecology, Shanghai Institutes of Biological Sciences, Chinese Academy of Sciences, Shanghai 200032, China; ${ }^{2}$ Department of Horticulture and Landscape Architecture, Purdue University, West Lafayette, IN 47906, USA; ${ }^{3}$ Key Laboratory of Plant Germplasm Enhancement and Specialty Agriculture, Wuhan Botanical Garden, Chinese Academy of Sciences, Wuhan, Hubei 430074, China; ${ }^{4}$ School of Life Sciences, University of Science and Technology of China, Hefei, Anhui 230026, China, ${ }^{5}$ Division of Plant Physiology, Indian Agricultural Research Institute, New Delhi 110012, India

Proteins in the PYR/PYL/RCAR family (PYLs) are known as receptors for the phytohormone ABA. Upon ABA binding, PYL adopts a conformation that allows it to interact with and inhibit clade A protein phosphatase 2Cs (PP2Cs), which are known as the co-receptors for ABA. Inhibition of the PP2Cs then leads to the activation of the SnRK2 family protein kinases that phosphorylate and activate downstream effectors in ABA response pathways. The PYL family has 14 members in Arabidopsis, 13 of which have been demonstrated to function as ABA receptors. The function of PYL13, a divergent member of the family, has been enigmatic. We report here that PYL13 differs from the other PYLs in three key residues that affect ABA perception, and mutations in these three residues can convert PYL13 into a partially functional ABA receptor. Transgenic plants overexpressing PYL13 show increased ABA sensitivity in seed germination and postgermination seedling establishment as well as decreased stomatal conductance, increased water-use efficiency, accelerated stress-responsive gene expression, and enhanced drought resistance. pyl13 mutant plants are less sensitive to ABA inhibition of postgermination seedling establishment. PYL13 interacts with and inhibits some members of clade A PP2Cs (PP2CA in particular) in an ABA-independent manner. PYL13 also interacts with the other PYLs and antagonizes their function as ABA receptors. Our results show that PYL13 is not an $A B A$ receptor but can modulate the ABA pathway by interacting with and inhibiting both the PYL receptors and the PP2C co-receptors.

Keywords: abiotic stress; drought; stress resistance; PYL; PP2C; SnRK2

Cell Research (2013) 23:1380-1395. doi:10.1038/cr.2013.149; published online 5 November 2013

\section{Introduction}

$\mathrm{ABA}$ is an important phytohormone that participates in plant abiotic stress signaling [1]. The PYR/PYL/ RCAR family proteins (hereafter referred to as PYLs) are ABA receptors in the cytoplasm and nucleus [2-5]. The PYL family has 14 members in Arabidopsis, including PYR1 and PYLs 1-13, each containing a START

*These two authors contributed equally to this work.

Correspondence: Jian-Kang Zhu

E-mail: jkzhu@purdue.edu

Received 24 June 2013; revised 11 September 2013; accepted 17 September 2013; published online 5 November 2013 domain. Several of the PYLs have been shown to bind and inhibit clade A protein phosphatase type 2Cs (PP2Cs, comprising ABI1, ABI2, HAB1, HAB2, AHG1, PP2CA, HAI1, HAI2, and HAI3) in the presence of ABA [2-4, 6, 7]. Structural studies confirmed that the PYLs are ABA receptors and showed that the $\mathrm{PP} 2 \mathrm{Cs}$ can function as coreceptors because they enhance the ABA-binding affinities of the PYLs [5, 8-11]. In Arabidopsis protoplasts, PYR1 and PYLs 1-12 could release ABI1 inhibition of ABA-dependent activation of $R D 29 B-L U C$ expression by the SnRK2 protein kinases such as OST1/SnRK2.6 [4]. Structural analysis revealed that the interaction between SnRK2.6 and HAB1 mimics the interaction between ABA-bound PYL2 and HAB1 [12]. PYLs can inhibit 
PP2C activity in the presence of ABA, and thereby activating SnRK2.6, which can phosphorylate slow anion channel1 (SLAC1) and activate SLAC1-mediated ion currents $[13,14]$.

PYLs have an ABA-binding pocket that comprises four highly conserved regions named CLs 1-4 [8]. The structure of PYL changes after ABA binding [5, 8-10], allowing the formation of an ABA-PYL-PP2C complex and the inhibition of PP2Cs, thus releasing the inhibition of SnRK2.2/2.3/2.6 by clade A PP2Cs $[15,16]$. SnRK2.2/2.3/2.6 are positive regulators of ABA signaling [17-19]. Activated SnRK2.2/2.3/2.6 can phosphorylate and activate transcription factors such as ABFs/ AREBs $[20,21]$ to induce the expression of ABAresponsive genes such as $R D 29 B$ [4]. In guard cells, the SnRK2s can also phosphorylate and activate the NADPH oxidase catalytic subunit RBOHF, leading to the production of reactive oxygen species (ROS) [17, 22, 23]. SnRK2s also interact with and phosphorylate plasma membrane anion channels such as SLAC1 to regulate ion transport [13, 24-26].

Many monomeric PYL proteins may also interact with certain PP2Cs in an ABA-independent manner in yeast two-hybrid assays and pull-down assays [2, 27]. However, this interaction is weak and in the particular case of PYL10-ABI1 interaction, the dissociation constant $(K \mathrm{~d})$ changed from $1.2 \mu \mathrm{M}$ without $\mathrm{ABA}$ to 0.02 $\mu \mathrm{M}$ with $\mathrm{ABA}$ [27], indicating that the interaction between PYL10 and ABI1 is enhanced by ABA. In vitro assays demonstrated that PYL10 shows obvious ABAindependent inhibition of ABI1, HAB1, and HAB2 with a PYL:PP2C ratio of 1:1, and PYLs 5-10 (except the untested PYL7) shows ABA-independent inhibition of ABI1, HAB1, HAB2, and PP2CA to different degrees with the PYL:PP2C ratio of 10:1 and 100:1 [27]. However, the ABA-independent inhibitions are much weaker than the ABA-dependent ones.

Although the PYL family proteins have been studied intensively in recent years [6, 28-31], little information is available for PYL13, which differs from other PYLs in the highly conserved regions of their ABA-binding pocket. To study the function of PYL13, we examined the interaction of PYL13 with clade A PP2Cs and the effects of PYL13 on the PP2Cs both in vitro and in vivo. We found that PYL13 can bind to the PP2Cs and in particular can constitutively inhibit PP2CA in an ABA-independent manner. Very interestingly, PYL13 interacts with the other PYLs and can reduce the ability of other PYLs to inhibit PP2Cs. Transgenic plants overexpressing PYL13 exhibit improved drought resistance and increased sensitivity to ABA inhibition of germination and postgermination seedling establishment while pyll3 mutant plants are less sensitive to ABA inhibition of postgermination seedling establishment, indicating that PYL13 is functionally important in planta.

\section{Results}

PYL13 differs from the other PYLs and does not function as an $A B A$ receptor

The PYL family contains 14 members. Although previous studies suggested that many are $\mathrm{ABA}$ receptors and have redundancy $[2,31]$, they also showed functional differences $[6,27]$. In the presence of ABA, PYR1 and PYLs 1-12 reduced the activity of ABI1 and activated SnRK2.6 to induce RD29B-LUC expression in Arabidopsis mesophyll protoplasts [4]. To test whether PYL13 can act as an ABA receptor, we reconstituted the ABA signaling pathway in wild-type (WT) protoplasts. As expected, the addition of PYL2 inhibited ABI1 activity and enabled the expression of ABA-dependent induction of RD29B-LUC (Figure 1A). However, co-transfection of PYL13 with ABI1, SnRK2.6, and ABF2 did not enable the expression of $R D 29 B-L U C$ (Figure 1A). The results suggest that PYL13 is a unique member of the PYL family and cannot function as an $\mathrm{ABA}$ receptor.

As noted earlier, the ABA-binding pocket in PYLs consists of four highly conserved regions named CLs 1-4 [8]. Amino acid sequence alignment shows that PYL13 differs from other PYLs in key residues in CLs 1-2 and CL4 (Q38 in CL1, F71 in CL2, and T135 in CL4; Supplementary information, Figure S1). To test whether these sequence variations may explain the functional difference of PYL13, we introduced point mutations (Q38K, F71L and T135N) to PYL13 to mimic the other PYLs, and transfected these mutated PYL13 together with ABI1, SnRK2.6, and ABF2 into protoplasts. None of the single mutations in PYL13 could mimic PYR1 in elevating the $R D 29 B-L U C$ expression. Double mutations (Q38K/F71L), and triple mutations (Q38K/F71L/T135N) of PYL13 could partially confer an ABA-dependent induction of $R D 29 B-L U C$ expression (Figure 1B). Q38 and F71 in CLs 1-2 appear to be the most important residues because PYL13 with these two residues mutated became largely functional in the reconstitution assay. The T135N mutation in CL4 could enhance the ability of PYL13 mutants to inhibit ABI1 activity in the presence of ABA. These results suggest that the inability of PYL13 to act as an ABA receptor is partially attributed to the three amino acid variations (Q38 in CL1, F71 in CL2, and T135 in CL4). However, as triple mutations (Q38K/F71L/T135N) of PYL13 cannot be as effective as PYR1 and PYL2 in inducing $R D 29 B-L U C$ expression, other sequence variations may also contribute to the inability of PYL13 to act 
A

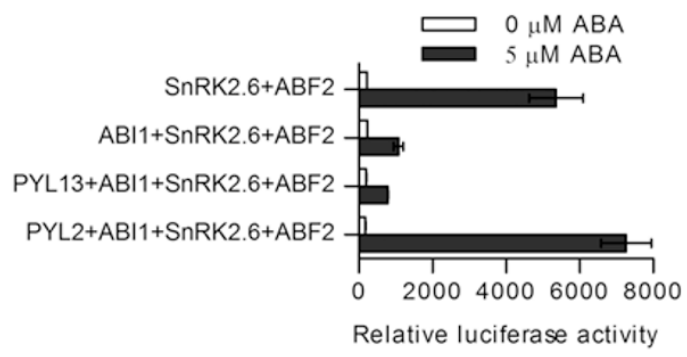

B

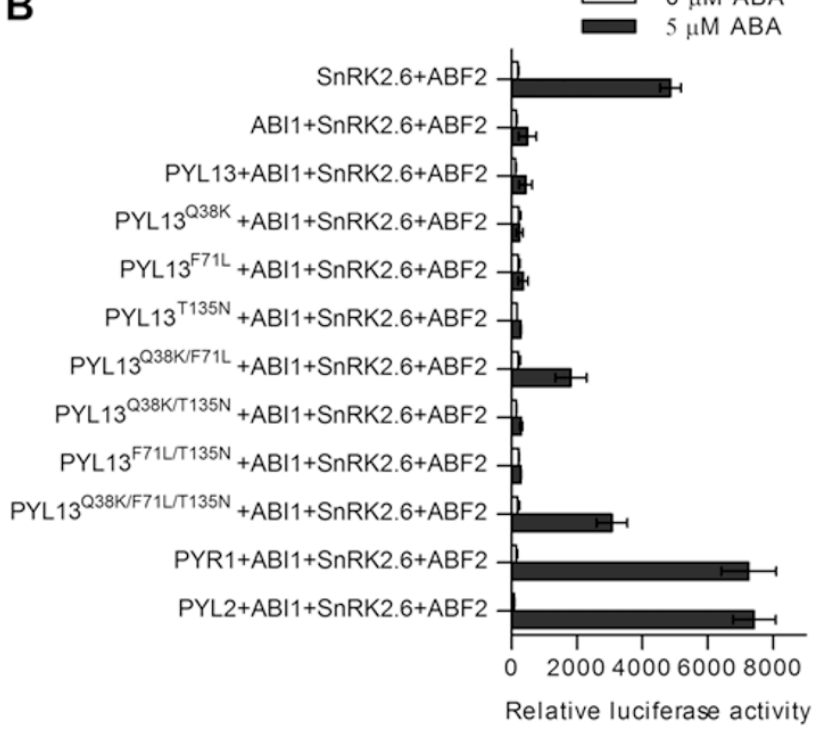

Figure 1 PYL13 differs from other PYLs. (A) PYL13 cannot antagonize the ability of $A B I 1$ to inhibit the ABA-dependent induction of $R D 29 B-L U C$ expression in protoplasts. The ABA signaling pathway was reconstituted by co-expression of PYLs, ABI1, SnRK2.6, and ABF2. (B) PYL13 mutants can mimic PYR1/PYL2 in elevating the expression of RD29B-LUC in protoplasts. WT Col-0 protoplasts were used in both $\mathbf{A}$ and $\mathbf{B}$. RD29B-LUC was used as the ABA-responsive reporter. ZmUBQ-GUS was used as the internal control. After transfection, protoplasts were incubated for $4.5 \mathrm{~h}$ under light in the absence of ABA (open bars) or in the presence of $5 \mu \mathrm{M}$ ABA (closed bars). Error bars indicate $\operatorname{SEM}(n=3)$.

fully as an $\mathrm{ABA}$ receptor.

\section{Overexpression of PYL13 confers drought resistance in Arabidopsis}

Previous studies showed that overexpression of PYL5 leads to an enhanced response to ABA and drought resistance [32]. Given that PYL13 does not function as an ABA receptor, we wonder whether it may still have a function in plants. We generated PYL13 overexpression (OE-PYL13) lines, and chose three homozygous T3 lines for phenotypic analysis. Northern blot analysis indicated that PYL13 transcripts were obviously more abundant in OE-PYL13 lines than in WT (Supplementary information, Figure S2).

OE-PYL13 lines exhibited an improved drought resistance (Figure 2A). After a 24-day drought treatment in soil, $>80 \%$ of $O E-P Y L 13$ transgenic plants survived and $<20 \%$ of WT plants survived (Figure 2B). The greater survival under drought stress of $O E-P Y L 13$ lines was associated with reduced water loss from leaves of the $O E$ PYL13 lines. Water loss from rosette leaves detached at the same developmental stage was slower in OE-PYL13 lines than in WT (Figure 2C). To characterize the drought resistance in $O E-P Y L 13$ transgenic plants, we determined several physiological parameters. When water was withheld for 5 days, transgenic plants overexpressing PYL13 showed a lower transpiration rate than WT plants (Figure 2D) and this was accompanied with a reduced stomatal conductance (Figure 2E). The photosynthetic rate (Figure $2 \mathrm{~F}$ ) and water-use efficiency (Figure 2G) were greater in OE-PYL13 lines than in WT. These results demonstrate that overexpression of PYL13 confers drought resistance in Arabidopsis.

Overexpression of PYL13 promotes stress-responsive gene expression

To further characterize the PYL13 overexpression lines, we tested the expression of stress-responsive genes including RD29A, KIN1, COR15A, and RAB18 in the transgenic plants (Figure $2 \mathrm{H}$ ). Under the unstressed control condition, the transcript levels of the four tested stress-responsive genes were 2-6-fold higher in the $O E-P Y L 13$ lines than in WT Col-0. Expression of RD29A, KIN1, COR15A, and $R A B 18$ was induced by ABA treatment in the $O E-P Y L 13$ transgenic lines as well as in Col-0. However, the levels of RD29A, KIN1, and COR15A were higher in OE-PYL13 seedlings than in Col-0 seedlings at early time points (e.g., 20 and $40 \mathrm{~min}$ ) after ABA treatment. At later time points (e.g., 6 h), the levels of RD29A, KIN1, COR15A, and $R A B 18$ in $O E-P Y L 13$ seedlings were similar to those in Col-0 seedlings.

We also tested the expression of stress-responsive genes in PYL13 overexpression lines under a lower concentration of ABA. Expression of RD29B, RAB18, P5CS1, and $R D 22$ was induced by $1 \mu \mathrm{M} \mathrm{ABA}$ treatment both in the $O E-P Y L 13$ transgenic lines and in Col-0 WT plants (Figure 2I), while the expression of RD29A, KIN1, and COR15A was not obviously induced by $1 \mu \mathrm{M}$ ABA treatment (data not shown). After $14 \mathrm{~h}$ treatment with $1 \mu \mathrm{M} \mathrm{ABA}$, the transcript levels of $R D 29 B, R A B 18, P 5 C S 1$, and $R D 22$ were 2-7-fold higher in the OE-PYL13 lines than in the Col-0 WT plants.

Taken together, these results suggested that overex- 

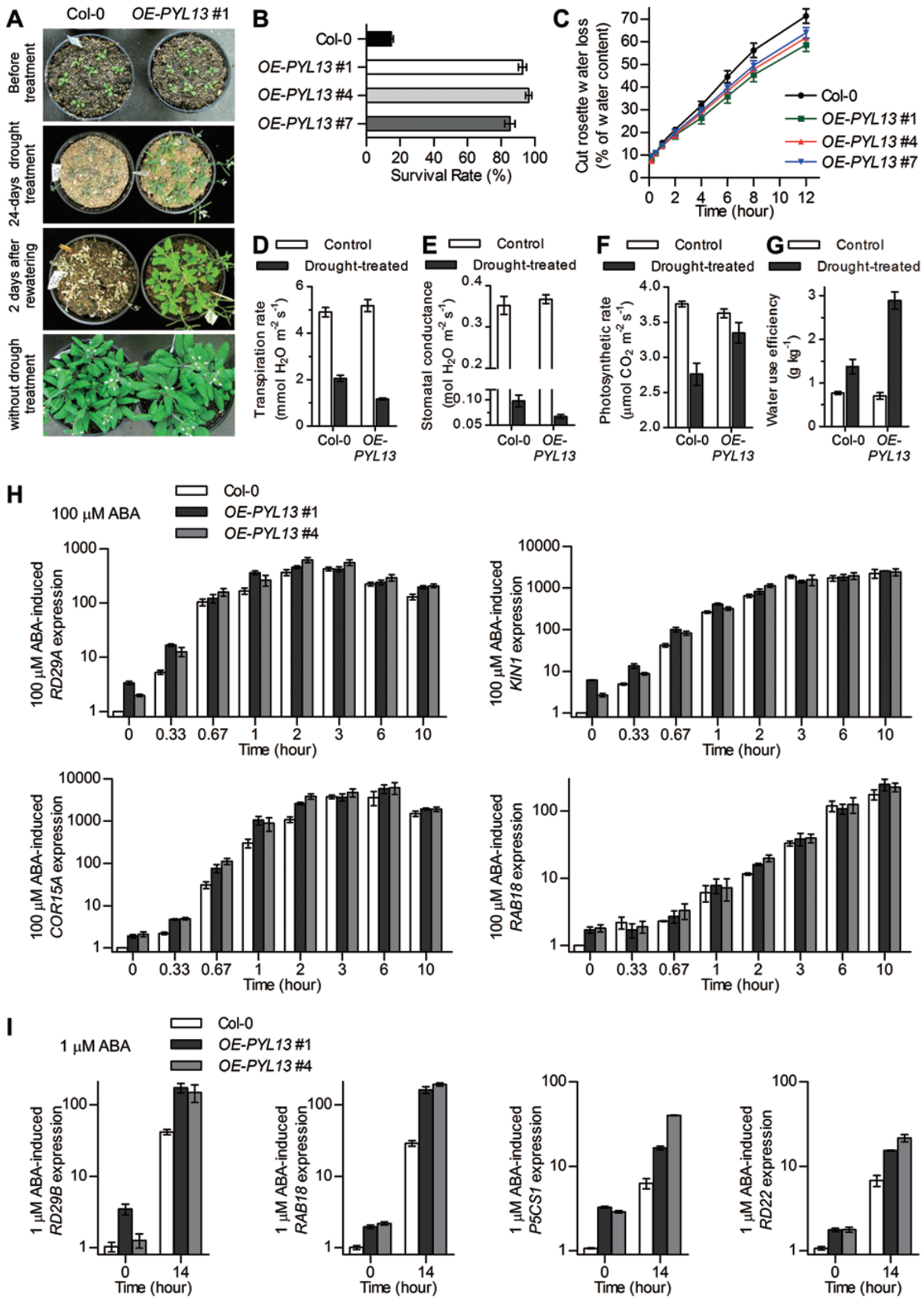
Figure 2 Overexpression of PYL13 confers drought resistance. (A) Fourteen-day-old plants were subjected to drought stress treatment (water was withheld for 24 days) before watering was resumed. Representative photographs show plants before drought treatment, after drought treatment, and without drought treatment. For the "after drought treatment", plants were also photographed 2 days after watering was resumed. (B) Surviving plants were counted 2 days after watering was resumed. Error bars indicate SEM $(n=3)$. (C) Cumulative transpirational water loss from rosettes of the WT Col-0 and PYL13 overexpression (OE-PYL13) lines at the indicated times after detachment. Error bars indicate SEM $(n=3)$. (D-G) Physiological parameters of OE-PYL13 lines under drought-stress conditions. Three-week-old plants were subjected to drought stress (water was withheld for 5 days) before parameters were measured with an infrared gas analyzer. Overexpression of $P Y L 13$ reduced the transpiration rate (D), reduced stomatal conductance $(E)$, increased the photosynthetic rate $(F)$, and increased wateruse efficiency (G). OE-PYL13 values are the means of three independent transgenic lines. Error bars indicate SEM $(n=4)$. (H) Expression of stress-responsive genes in OE-PYL13 lines. Quantitative RT-PCR was conducted on 10-day-old seedlings with $100 \mu \mathrm{M}$ ABA treatment for $0 \mathrm{~min}, 20 \mathrm{~min}, 40 \mathrm{~min}, 1 \mathrm{~h}, 2 \mathrm{~h}, 3 \mathrm{~h}, 6 \mathrm{~h}$, and $10 \mathrm{~h}$. The expression levels of RD29A, KIN1, COR15A and RAB18 in Col-0 before ABA treatment were set as 1. Error bars indicate SEM $(n=3)$. (I) Expression of stressresponsive genes in OE-PYL13 lines. Quantitative RT-PCR was conducted on 10-day-old seedlings with $1 \mu \mathrm{M}$ ABA treatment for $14 \mathrm{~h}$. The expression levels of $R D 29 B, R A B 18, P 5 C S 1$ and $R D 22$ in Col-0 before ABA treatment were set as 1 . Error bars indicate SEM $(n=3)$.

pression of PYL13 enhances stress-responsive gene expression in plants.

PYL13 interacts with clade A PP2Cs in an ABA-independent manner

To investigate how PYL13 may function in plants, we examined the interaction between PYL13 and clade A PP2Cs in yeast two-hybrid assays. The results show that PYL13 binds to all PP2Cs tested in an ABA-independent manner (Figure 3A). PYL13 binds strongly with ABI1, ABI2, PP2CA, and HAI2 but weakly with HAB1 and HAI1 (Figure 3A). We confirmed these results in pulldown assays with purified recombinant proteins, in which PYL13 interacted with all PP2Cs tested in an ABA-independent manner while PYR1 interacted with ABI1 in an ABA-dependent manner (Figure 3B). In the pull-down assay, PYL13 binding was much stronger with PP2CA than with AHG1, ABI1, or ABI2 (Figure 3B). To determine whether PYL13 and PP2Cs may interact in an ABA-independent manner in vivo, we co-expressed c-myc-tagged PYL13 and hemagglutinin (HA)-tagged PP2Cs in protoplasts. Co-immunoprecipitation (co-IP) assays using ABI1-HA and PP2CA-HA as baits captured c-myc-PYL13 in an ABA-independent manner (Figure 3C). The binding of PP2CA with PYL13 again appeared much stronger than that of ABI1 with PYL13. These results demonstrated that PYL13 constitutively binds to clade A PP2Cs, but the binding intensity differs among members of clade A PP2Cs.

PYL13 differentially inhibits clade A PP2Cs in an ABAindependent manner and is important for seed germination

PYL13 interacts with all members of clade A PP2Cs tested but binds most strongly to PP2CA (Figure 3). To investigate whether PYL13 inhibits PP2Cs in planta, we co-expressed PYL13 with all clade A PP2Cs in WT protoplasts. Transfections of all PP2Cs together with SnRK2.6 and ABF2 inhibited RD29B-LUC expression to different degrees (Figure $4 \mathrm{~A}$ ), and the degree of inhibition was correlated with the intensity of interaction between PP2Cs and SnRK2.6 [4]. For example, the intensities of PP2C interaction with SnRK2.6 were $\mathrm{ABI} 1>\mathrm{HAB} 1>\mathrm{ABI} 2$ in yeast two-hybrid assays, and the inhibition of $R D 29 B-L U C$ expression was greater for $\mathrm{ABI} 1$ than $\mathrm{HAB} 1$ and was greater for $\mathrm{HAB} 1$ than for ABI2 in the protoplast experiments (Figure 4A). Co-transfections of PYL13 together with ABI1, ABI2, HAB1, HAB2, or AHG1 did not enable RD29B-LUC expression, but co-transfections of PYL13 with PP2CA, HAI2, or HAI3 enabled the ABA-dependent induction of RD29B-LUC expression (Figure 4A). In the control experiments, co-transfections of PYR1 and PYL2 with ABI1 restored ABA-induced RD29B-LUC expression (Figure 4A). These results indicated that PYL13 inhibits the phosphatase activities of PP2CA, HAI2, and HAI3. The ABA responsiveness of the PYL13-transfected protoplasts as well as of the protoplasts without any transfected PYLs was presumably due to the activities of endogenous PYLs present in the protoplasts.

To investigate the underlying mechanism by which PYL13 differentially inhibits the function of PP2Cs, we examined the effects of recombinant PYL13 on the phosphatase activities of recombinant PP2Cs with and without ABA. The results showed that PYL13 acts as a strong constitutive inhibitor of PP2CA phosphatase activity and a weak inhibitor of ABI1 activity but has almost no inhibitory activity against ABI2 at the molar ratio of 1:1 (Figure 4B). The inhibition by PYL13 is ABAindependent, whereas the inhibition by PYR1 and PYL2 

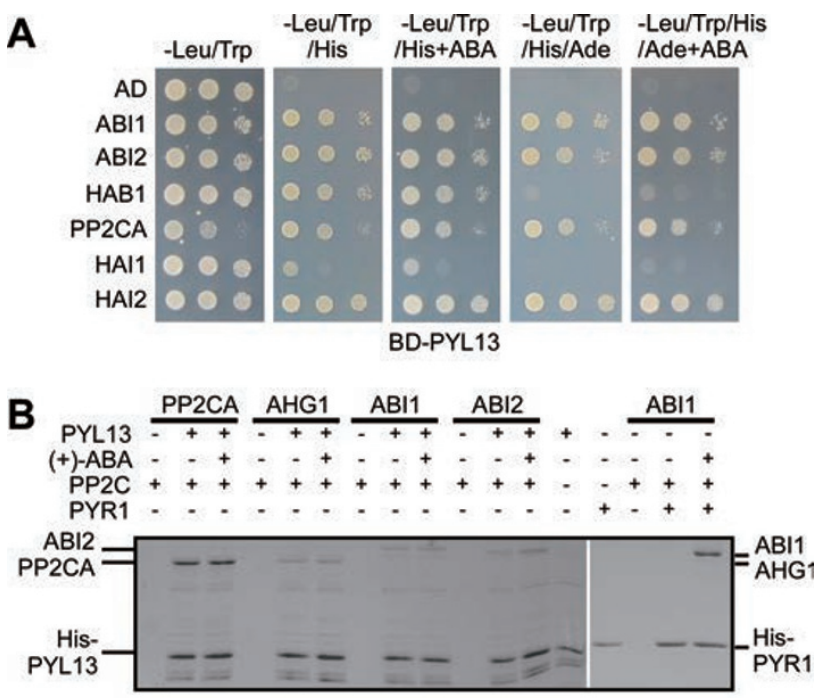

C

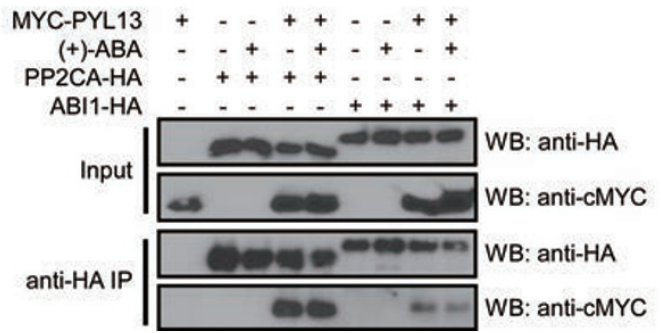

Figure 3 PYL13 binds to clade A PP2Cs in an ABA-independent manner. (A) PYL13 interacts with clade A PP2Cs in a yeast twohybrid assay. PYL13 fused to the GAL4-DNA-binding domain (BD) was used as prey, and PP2Cs fused to the GAL4-activating domain $(A D)$ were used as baits. The interaction was determined by growth assay on media lacking $\mathrm{His}$ and His/Ade in the presence and absence of $10 \mu \mathrm{M}$ ABA. Dilutions $\left(10^{-1}, 10^{-2}\right.$, and $10^{-3}$ ) of saturated cultures were spotted onto the plates, which were photographed after 5 days. (B) Recombinant PYL13 binds to clade A PP2Cs in an ABA-independent manner. His-tagged PYL13 ( 22 kD) and PYR1 ( 25 kD) were used to pull down PP2Cs without tag. PYL13 interacted with PP2CA, AHG1, ABI1 and $A B I 2$ independent of $A B A$ while PYR1 interacted with $A B I 1$ in the presence of $200 \mu \mathrm{M}$ ABA. (C) ABA-independent binding of PYL13 with PP2CA and ABI1 in protoplasts. Total soluble proteins (input) were extracted from Arabidopsis protoplasts that transiently expressed the indicated constructs. PP2C-interacting proteins were immunoprecipitated with anti-HA mouse antibodies and detected with anti-HA and anti-cMYC rabbit antibodies.

is ABA-induced (Figure 4B). PYL13 reduced PP2CA phosphatase activity to $55 \% \pm 5 \%$ without $\mathrm{ABA}$ and to $54 \% \pm 5 \%$ with $1.25 \mu \mathrm{M} \mathrm{ABA}$, and reduced ABI1 phosphatase activity to $75 \% \pm 3 \%$ without $\mathrm{ABA}$ and to $75 \% \pm$ $3 \%$ with $1.25 \mu \mathrm{M}$ ABA. In contrast, PYL13 only reduced $\mathrm{ABI} 2$ phosphatase activity to $89 \% \pm 8 \%$ without $\mathrm{ABA}$ and to $93 \% \pm 6 \%$ with $1.25 \mu \mathrm{M} \mathrm{ABA}$, and this level of activity did not differ from that in the buffer control with ABA $(93 \% \pm 5 \%)$. These results show that PYL13 differentially inhibits the phosphatase activities of PP2Cs in an ABA-independent manner.

We observed that the basal RD29B-LUC expression level was elevated in protoplasts co-transfected with PP2CA-PYL13, HAI2-PYL13, or HAI3-PYL13, which is consistent with PYL13 inhibition of these PP2Cs in an ABA-independent manner in vivo (Figure 4A). To exclude interference by endogenous PP2Cs in WT protoplasts and to assess the ABA-independent selective inhibition of PP2Cs by PYL13, we co-expressed PYL13 with PP2CA, HAI1, HAI2, and HAI3 in abil/hab1/ abi2 triple mutant protoplasts. This mutant is deficient in ABI1, HAB1, and ABI2 but does not show strong constitutive activation of SnRK2.2/2.3/2.6 [4, 33]. Expression of $R D 29 B-L U C$ in the absence of ABA was increased in the co-transfections of PP2CA-PYL13, HAI2-PYL13, or HAI3-PYL13 together with SnRK2.6 and ABF2 but an increase was not observed in the control transfections of PYL13-HAI1 or PYL2-PP2CA (Figure 4C). The triple mutation Q38K/F71L/T135N abolished the ability of PYL13 to elevate $R D 29 B-L U C$ expression in the absence of $A B A$, indicating that the three amino acid variations are responsible for the ABA-independent inhibition of PP2Cs by PYL13 (Figure 4C). These data further suggested that PYL13 inhibits PP2CA, HAI2, and HAI3 in an ABA-independent manner in vivo.

Overexpression of AtPP2CA resulted in ABA insensitivity while knockout of AtPP2CA caused ABA hypersensitivity during seed germination [34]. Given the strong inhibition by PYL13 on PP2CA activity in vitro and in vivo, we investigated whether PP2CA function in planta may be impacted by PYL13 overexpression. We tested the seed germination of PYL13 overexpression lines on MS medium containing $0,0.5$ or $1 \mu \mathrm{M}$ ABA. In the absence of exogenous ABA, OE-PYL13 seeds germinated as well as WT seeds (Figure 4E, left panel). In the presence of $0.5 \mu \mathrm{M}$ ABA, the germination of OE-PYL13 \#1 was greatly inhibited by ABA (Figure 4E, middle

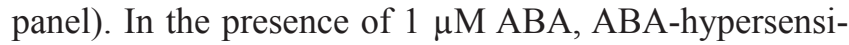
tive inhibition of seed germination was observed for $O E$ PYL13 \#1 and OE-PYL13 \#7 (Figure 4E, right panel). Postgermination seedling establishment inhibition in the presense of $0.2 \mu \mathrm{M}$ ABA was observed for OE-PYL13 \#1 (Figure 4F). The germination sensitivity to ABA was correlated with the expression level of PYL13 (Supplementary information, Figure S2). In contrast, pyl13-1 (SALK 041176) and pyl13-2 (SALK_071488) mutants (Figure 4D) were less sensitive to ABA inhibition of postgermiantion seedling establishment (Figure 4F) and showed no obvious phenotype upon ABA inhibition of 
A

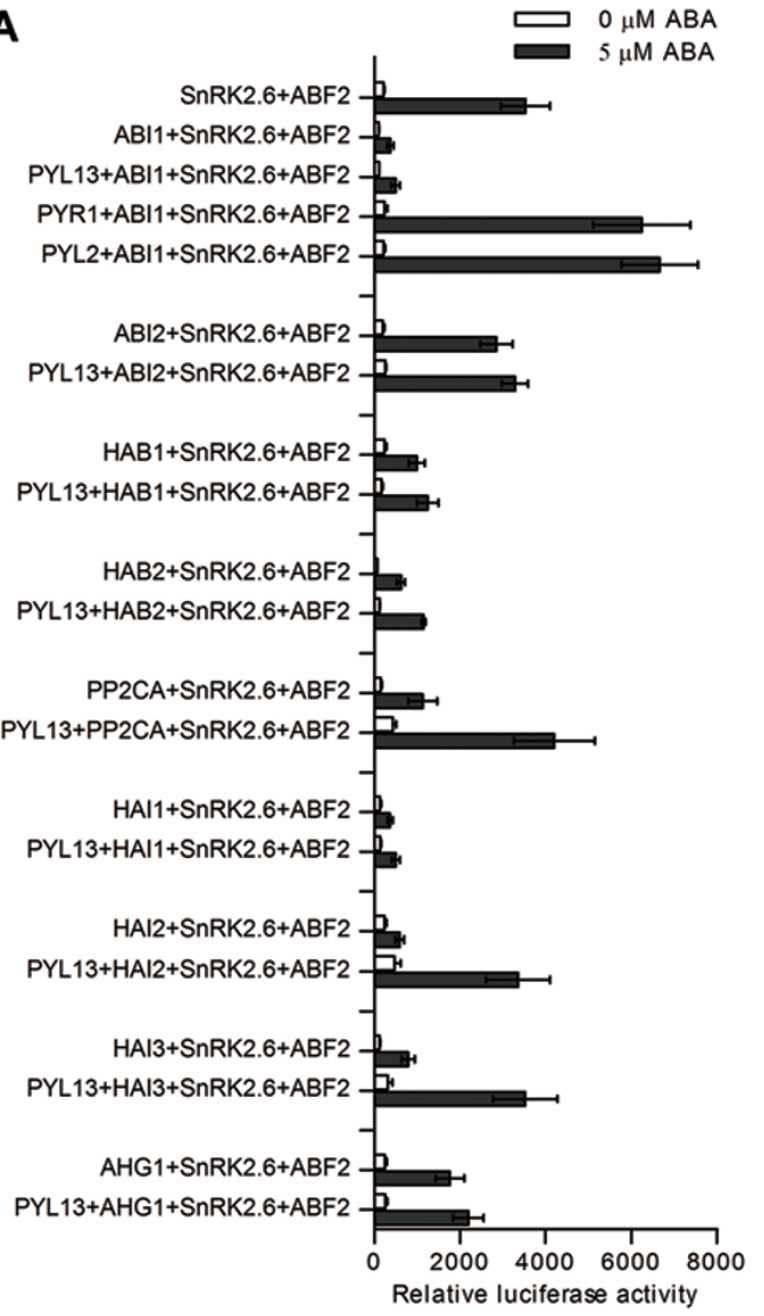
ABI1+SnRK2.6+ABF2 PYL13+AB11+SnRK2.6+ABF2 PYR1+AB11+SnRK2.6+ABF2 PYL2+ABI1+SnRK2 6+ABF2 $\mathrm{ABI} 2+\mathrm{SnRK} 2.6+\mathrm{ABF} 2-$ HAB1+SnRK2.6+ABF2 PYL13+HAB1+SnRK2.6+ABF2 HAB2+SnRK2.6+ABF2 PYL13+HAB2+SnRK2.6+ABF2 PYL13+PP2CA+SnRK2.6+ABF2 HAl1+SnRK2.6+ABF2 PYL13+HAl1+SnRK2.6+ABF2 -

B

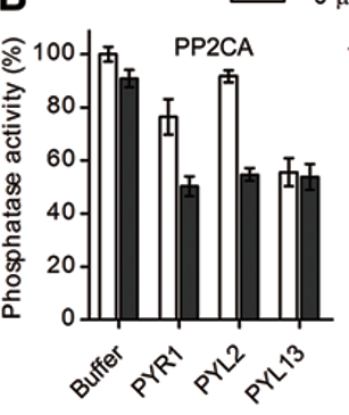

$$
0 \mu \mathrm{MABA}
$$
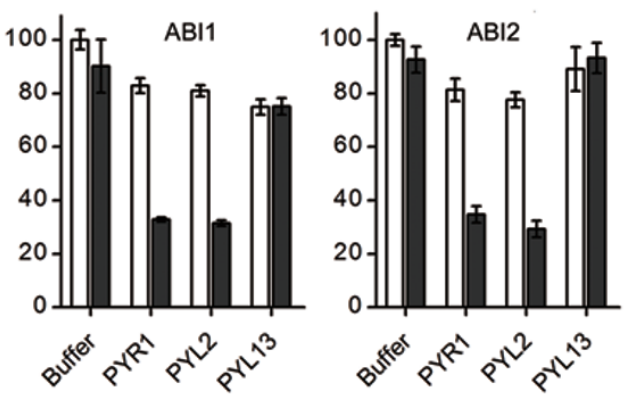

C

PYL13
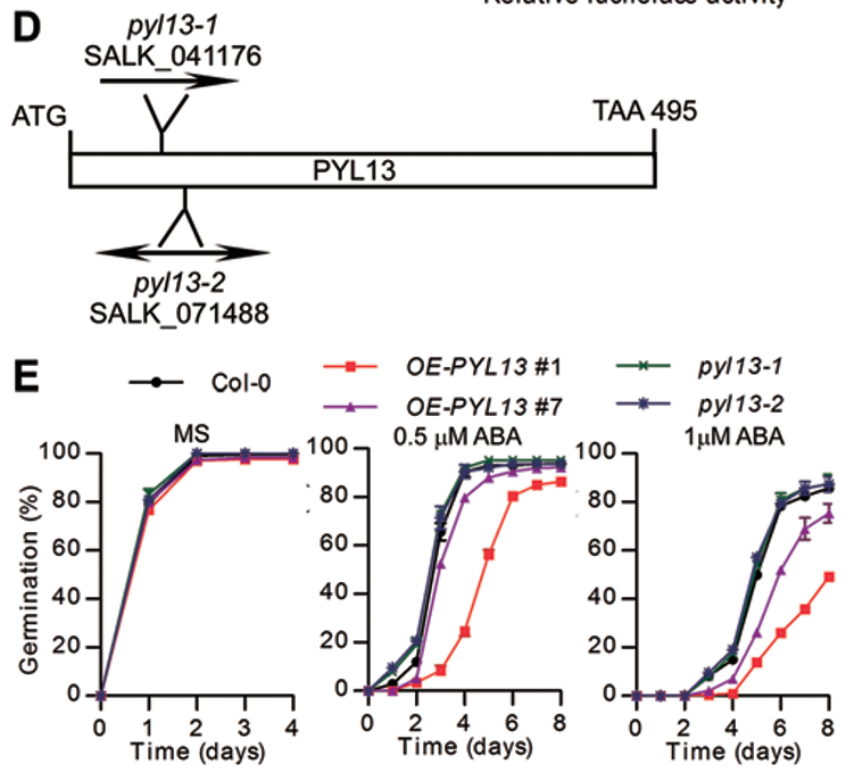

F $\mathrm{COl}_{\text {pyl }} \rightleftharpoons$ pyl13-2

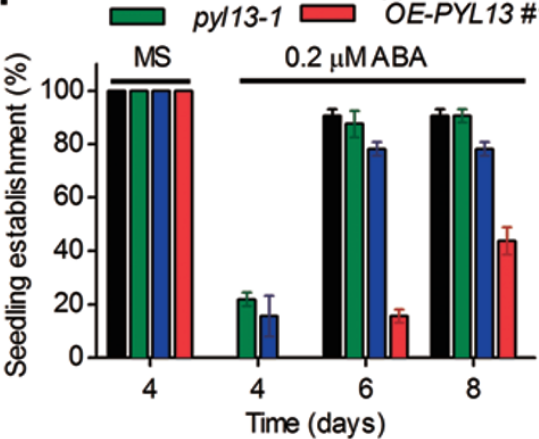

germination (Figure 4E).

PYL13 interacts with and antagonizes other PYLs

To further investigate the molecular mechanism of PYL13 function in plants, we generated transgenic Arabidopsis plants expressing tagged PYL13 under the native PYL13 promoter (ProPYL13: PYL13-HA-YFP
(Supplementary information, Figure S3A) and purified PYL13-associated proteins using anti-GFP-agarose in extracts of floral organs of the transgenic plants (Supplementary information, Figure S3B). PYL13-associated proteins were identified by mass spectrometric analyses (Supplementary information, Table S1). The associated proteins included not only PP2CA and HAI1 but also 
Figure 4 PYL13 inhibits clade A PP2Cs in an ABA-independent manner. (A) Inhibition of RD29B-LUC expression by PP2CA, $\mathrm{HAI} 2$, and HAI3 was antagonized by PYL13 in WT protoplasts. ABA signaling pathway was reconstituted by co-expression of PYLs, PP2Cs, SnRK2.6, and ABF2. RD29B-LUC was used as the ABA-responsive reporter. ZmUBQ-GUS was used as the internal control. After transfection, protoplasts were incubated for $4.5 \mathrm{~h}$ under light in the absence of $A B A$ (open bars) or in the presence of $5 \mu \mathrm{M}$ ABA (closed bars). Error bars indicate SEM $(n=9)$. (B) The activities of recombinant clade A PP2Cs were differentially inhibited by recombinant PYL13 independently of ABA. The phosphatase activities of PP2CA, ABI1, and ABI2 were measured in the absence or presence of ABA $(1.25 \mu \mathrm{M})$, and with or without PYLs. The concentration of PP2Cs and PYLs was $0.3 \mu \mathrm{M}$. The $A_{405}$ value of reaction without PP2C was set as $0 \%$ and that of the reaction with PP2C but without $A B A$ and PYLs was set as $100 \%$. Error bars indicate SEM ( $n=6$ for PP2CA and ABI2, and $n=3$ for ABI1). (C) ABA-independent induction of RD29B-LUC in abi1/hab1/abi2 protoplasts that transiently expressed PYL13, PP2CA, HAI2, or HAI3. After transfection, protoplasts were incubated for $4.5 \mathrm{~h}$ under light in the absence of ABA. Error bars indicate SEM ( $n=3)$. (D) Diagram of the PYL13 gene. The open box indicates exon. The two T-DNA insertions are also indicated. (E) Seed germination of the WT, pyl13 mutants and PYL13 overexpression (OE-PYL13) lines in MS medium and MS plus 0.5 or $1 \mu \mathrm{M}$ ABA. The panels show the percentage of seeds with emerged radicle. Error bars indicate SEM $(n=3)$. (F) Seedling establishment of the WT, pyl13 mutants and OE-PYL13 transgenic line in MS medium and MS plus $0.2 \mu \mathrm{M}$ ABA. The panels show the percentage of seeds that developed green cotyledons. Error bars indicate SEM $(n=3)$.

PYL2 and PYL9. While the result confirmed the interaction of PYL13 and PP2Cs in planta (Figure 3), it also raised the possibility of PYL13 interaction with and affecting the activities of other PYLs.

To confirm the interaction between PYL13 and PYL2/ PYL9 and to determine whether PYL13 may bind with other PYLs, we examined the interaction of PYL13 with PYLs in yeast two-hybrid assays. In this experiment, PYL13 interacted with PYR1, PYLs 1-4, PYL6, and PYLs 11-12 in an ABA-independent manner (Figure 5A). These results thus confirmed the interaction between PYL13 and PYL2 identified in immunoprecipitation and mass spectrometric experiments. However, we were unable to detect an interaction between PYL13 and PYL9, perhaps due to the cellular differences between yeast and Arabidopsis or due to indirect interactions between the two proteins in Arabidopsis.

To further characterize the interactions between PYL13 and PYLs in vivo, we investigated their interactions in protoplasts by the firefly luciferase (LUC) complementation assay [35]. We fused PYL13 to the C-terminal domain of LUC (PYL13-cLUC), and fused PYLs to the N-terminal domain of LUC (PYLs-nLUC), and co-expressed them in protoplasts. The relative LUC activities were measured, and the results suggested that PYL13 binds to all PYLs tested but with different intensities (Figure 5B). PYL13 binds more strongly to PYR1, PYLs 1-5, PYL7, and PYLs 10-11 than to PYL6, PYLs $8-9$, or PYL13 (Figure 5B). The direct interactions between PYL13 and two of the PYLs were also verified in pull-down assays with purified recombinant proteins. GST-tagged PYL1 ( 51 kD) and PYL2 ( 46 kD) were pulled down by His-tagged PYL13 ( 22 kD) in an ABAindependent manner (Figure 5C). Previous structural and biochemical investigations demonstrated that several
PYLs (such as PYR1, PYL1, PYL2, PYL3) form homodimers in solution and that the dimer formation is important in regulating $\mathrm{ABA}$ binding and $\mathrm{PP} 2 \mathrm{C}$ interactions $[5,8,11,27,28,36,37]$. Our results suggest that PYL13 can form heterodimers with other PYLs.

To test the functional significance of potential heterodimer formation between PYL13 and other PYLs, we compared the abilities of PYL1 and PYL2 and the protein complexes of PYL13-PYL1 and PYL13-PYL2 to inhibit the phosphatase activity of ABI1 using purified recombinant proteins and the synthetic phosphatase substrate p-nitrophenyl phosphate (pNPP). The results showed that inhibition of ABI1 in the presence of ABA was less for the PYL13-PYLs dimers than for the PYLs alone (Figure 5D). In the presence of $10 \mu \mathrm{M} \mathrm{ABA}$, ABI1 phosphatase activity was reduced to $67 \% \pm 6 \%$ by PYL1 but only to $88 \% \pm 8 \%$ by PYL13-PYL1, and was reduced to $50 \% \pm 3 \%$ by PYL2 but only to $78 \% \pm 10 \%$ by PYL13-PYL2. These results suggest that, by forming heterodimers, PYL13 can reduce the ability of PYL1 and PYL2 to inhibit the phosphatase activity of ABI1 in vitro.

To investigate the physiological significance of the binding between PYL13 and PYLs in vivo, we coexpressed PYL13 with one of the other PYLs, ABI1, SnRK2.6, and ABF2 in WT protoplasts. When the amount of transfected PYL13 plasmid was 2-fold of that of the other PYLs, co-transfections with PYL13 decreased the activity of the ABA-responsive PYLs (Figure 5E). The level of inhibition by PYL13 depended on the PYLs and ranged from $27 \%$ with PYL2 to $67 \%$ with PYL1 (Supplementary information, Figure S4). These results indicated that PYL13 can antagonize the activity of other PYLs in plants. PYL13 inhibition of other PYLs might result from direct interactions between PYL13 and other PYLs. 
A

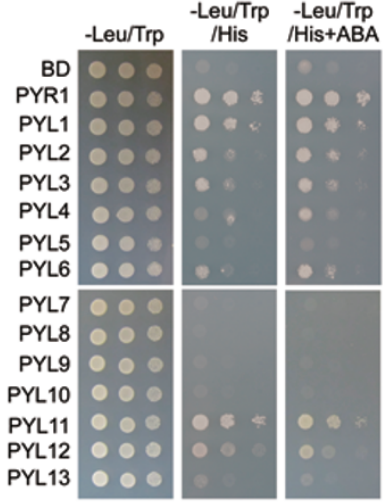

B

$$
\begin{array}{r}
n L U C+c L U C \\
n L U C+P Y L 13-c L U C
\end{array}
$$
PYR1-nLUC+PYL13-cLUC PYL1-nLUC+PYL13-cLUC PYL2-nLUC+PYL13-CLUC PYL3-nLUC+PYL13-cLUC PYL4-nLUC+PYL13-cLUC PYL5-nLUC+PYL13-cLUC PYL6-nLUC+PYL13-cLUC PYL7-nLUC+PYL13-cLUC PYL8-nLUC+PYL13-CLUC PYL9-nLUC+PYL13-cLUC PYL10-nLUC+PYL13-CLUC PYL11-nLUC+PYL13-cLUC PYL13-nLUC+PYL13-CLUC

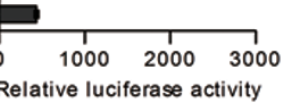

C

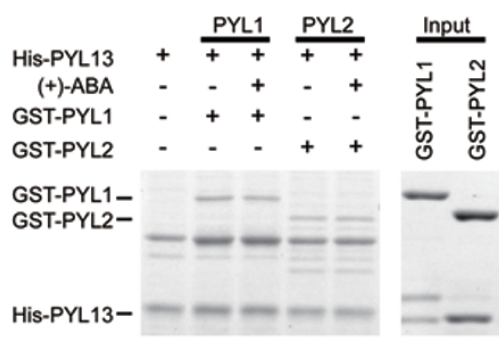

D

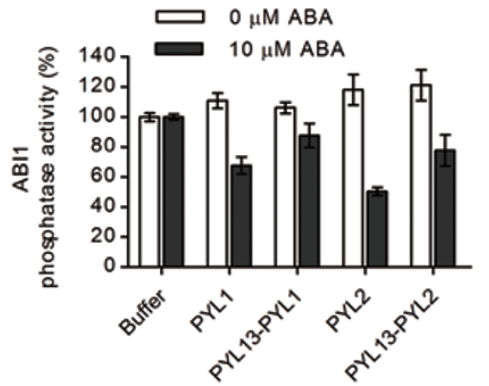

E

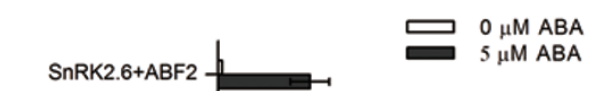

\section{XPYL13+P} $\mathrm{ABI} 1+\mathrm{SnRK} 2.6+\mathrm{ABF} 2$

PYL13+ABI1+SnRK2.6+ABF2 $2 \times P Y L 13+A B I 1+S n R K 2.6+A B F 2-$

PYR1+ABI1+SnRK2.6+ABF2 R1+ABI1+SnRK2.6+ABF2

PYL1+ABI1+SnRK2.6+ABF2

2xPYL13+PYL1+ABI1+SnRK2.6+ABF2

PYL2+AB11+SnRK2.6+ABF2 2xPYL13+PYL2+ABI1+SnRK2.6+ABF2

PYL3+ABI1+SnRK2.6+ABF2 2xPYL13+PYL3+ABI1+SnRK2.6+ABF2

PYL4+ABI1+SnRK2.6+ABF2 2xPYL13+PYL4+ABI1+SnRK2.6+ABF2

PYL5+ABI1+SnRK2.6+ABF2 2xPYL13+PYL5+ABI1+SnRK2.6+ABF2

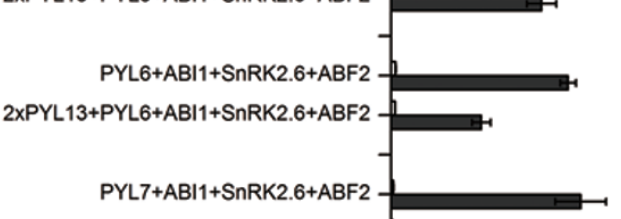
2xPYL13+PYL7+ABI1+SnRK2.6+ABF2

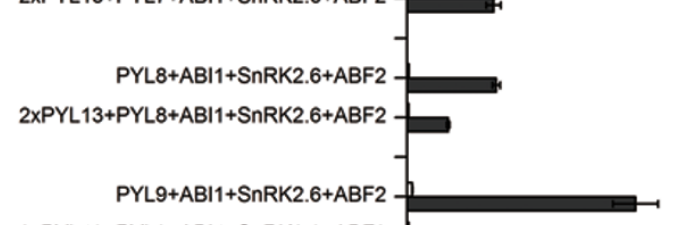
2xPYL13+PYLO+ABI1+SnRK2.6+A

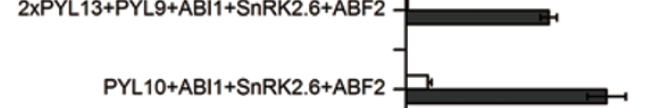
2xPYL13+PYL10+ABI1+SnRK2.6+ABF2 -

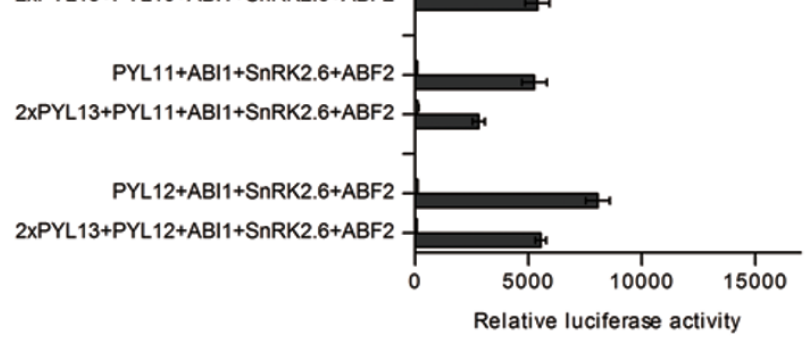

$\mathbf{F}$

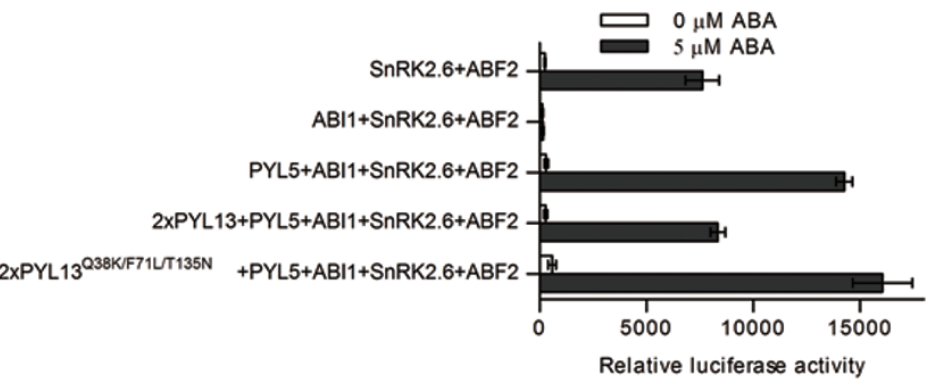

Cell Research | Vol 23 No 12 | December 2013 
Figure 5 PYL13 can antagonize the activities of other PYLs. (A) PYL13 interacts with other PYLs in yeast two-hybrid assays. PYL13 fused to the GAL4-activating domain $(A D)$ was used as prey, and PYLs fused to the GAL4-DNA binding domain (BD) were used as baits. The interaction was determined by growth assay on media lacking His and His/Ade in the presence and absence of $10 \mu \mathrm{M}$ ABA. Dilutions $\left(10^{-1}, 10^{-2}\right.$, and $\left.10^{-3}\right)$ of saturated cultures were spotted onto the plates, which were photographed after 5 days. (B) PYL13 interacts with all PYLs in firefly luciferase (LUC) complementation assays in protoplasts. After transfection, protoplasts were incubated overnight under light at room temperature, and the LUC activities in living protoplasts were measured. Error bars indicate SEM $(n=6)$. (C) Recombinant PYL13 binds to PYL1 and PYL2. His-tagged PYL13 (L13, 22 kD) was used to pull down GST-tagged PYL1 (L1, 51 kD) and PYL2 (L2, 46 kD). (D) PYL13 can reduce the ability of PYL1 and PYL2 to inhibit ABI1 phosphatase activity. The phosphatase activity of $A B I 1$ was measured (using pNPP) in absence or presence of $10 \mu \mathrm{M} A B A$, with or without PYLs, and with PYL13-PYL dimers. The concentration of ABI1 and PYLs was $0.3 \mu \mathrm{M}$. The $A_{405}$ value of reaction without $A B I 1$ was set as $0 \%$ and that of the reaction with $A B I 1$ but without ABA and PYLs was set as $100 \%$. Error bars indicate SEM $(n=6)$. (E) PYL13 can reduce the abilities of other PYLs to inhibit the ABI1 activity in WT (Col-0) protoplasts. The ABA signaling pathway was reconstituted by co-expression of PYL13, PYLs, $A B I 1$, SnRK2.6, and ABF2. The designation "2× PYL13" indicates that the concentration of PYL13 plasmids was 2-fold greater than that of PYL2 plasmids. ZmUBQ-GUS was used as the internal control. After transfection, protoplasts were incubated for $4.5 \mathrm{~h}$ under light in the absence of ABA (open bars) or in the presence of $5 \mu \mathrm{M}$ ABA (closed bars). Error bars indicate SEM ( $n$ = 3). (F) The PYL13 triple mutant Q38K/F71L/T135N cannot reduce the ability of PYL5 to inhibit the ABI1 activity in WT (Col-0) protoplasts. Error bars indicate SEM $(n=8)$.

To test the functional significance of the three special residues (Q38, F71, and T135) in PYL13 on its inhibition of other PYLs, we co-expressed PYL13 triple mutant Q38K/F71L/T135N with PYL5, ABI1, SnRK2.6, and ABF2 in WT protoplasts. The triple mutation Q38K/ F71L/T135N abolished the ability of PYL13 to antagonize the activity of PYL5 in protoplasts (Figure 5F). These results indicated that the three amino acid variations are responsible for the inhibition of other PYLs by PYL13.
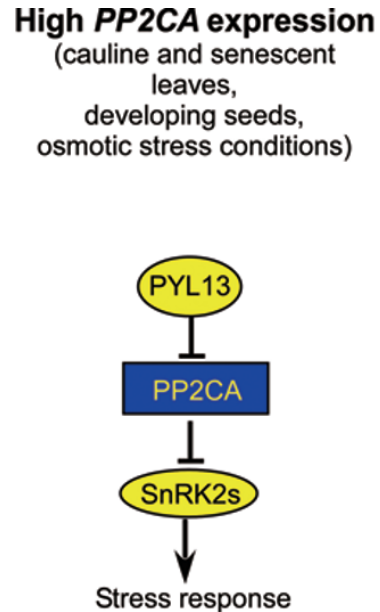

Figure 6 Model of the dual role of PYL13. PYL13 has dual roles in Arabidopsis. In tissues and conditions where PP2CA is highly expressed, PYL13 constitutively inhibits PP2CA activity (left panel). In tissues and conditions where PP2CA expression is low, PYL13 antagonizes other ABA-responsive PYLs via heterodimer formation (right panel).

\section{Discussion}

In this study, we found that PYL13 has dual roles in Arabidopsis (Figure 6). First, PYL13 inhibits PP2CA, HAI2 and HAI3, which activates ABA responses. Second, PYL13 can negatively regulate ABA responses by antagonizing ABA-responsive activities of other PYLs. PYL13 interacts with all members of tested clade A PP2Cs and many of the other members of PYLs in an ABA-independent manner. The interaction between PYL13 and PYLs is weaker than that between PYL13 and PP2Cs. PYL13 has a particularly strong interaction with PP2CA and preferentially inhibits PP2CA activity. $P P 2 C A$ is strongly expressed in cauline leaves and developing seeds, whereas it is only weakly expressed in rosette leaves and roots but the expression is upregulated by osmotic stress (http://bar.utoronto.ca/efp/cgi-bin/efpWeb.cgi). In conditions and tissues and developmental stages where PP2CA is prevalent, the primary function of PYL13 is likely to inhibit PP2CA to activate a subset of $\mathrm{ABA}$ responses. However, when PP2CA is not present at high levels, PYL13 would negatively regulate ABA responses by interacting with other PYLs. At present, it is unclear how PYL13 antagonizes other PYLs by interacting with them. It is possible that the interaction leads to interference with the ability of the other PYLs to bind $\mathrm{ABA}$ and/or to bind and inhibit downstream PP2Cs. This antagonistic role of PYL13 may be important in situations (e.g., very high ABA concentrations) where overactivation of ABA signaling needs to be prevented.

Yeast two-hybrid, pull-down, and co-IP assays demonstrated that PYL13 interacted with all clade A PP2Cs in an ABA-independent manner (Figure 3). Phosphatase activity assays showed that PYL13 strongly inhibited 
PP2CA in an ABA-independent manner (Figure 4B). The inhibitory mechanism of PYL13 differs from the one reported previously for PYLs 5-10, which also interact with and inhibit PP2C activity in the absence of ABA [2, 27, 32]. Although these PYLs have high basal activities, ABA can enhance their interaction with PP2Cs and their inhibition of PP2C phosphatase activity [27, 38]. PYL10 also forms stable complexes with ABI1 in the absence of ABA, however, the binding of PYL10 to ABI1 is much stronger in the presence than in the absence of ABA [27]. In our study, in contrast, interactions between PYL13 and PP2Cs were unaffected by ABA, i.e., these interactions are completely ABA-independent (Figures 3 and 4A). The inhibition of PP2CA phosphatase activity by PYL13 with or without ABA is indistinguishable from that by PYR1/PYL2 with ABA (Figure 4B). These results are supported by the reconstitution of ABA signaling in protoplasts and by the phenotypes of the PYL13 overexpression transgenic plants (Figure 4A, 4C and 4D).

PYL13 can inhibit PP2CA activity in vivo (Figure 4C). When PYL13 was co-transfected with PP2CA, HAI2, or HAI3 in abil/habl/abi2 protoplasts, the basal expression of $R D 29 B-L U C$ in the absence of $\mathrm{ABA}$ was enhanced (Figure 4C). The PYL13 triple mutant Q38K/ $\mathrm{F} 71 \mathrm{~L} / \mathrm{T} 135 \mathrm{~N}$ lost the ABA-independent induction of the RD29B-LUC basal expression (Figure 4C), suggesting that it is these sequence variations that confer $\mathrm{ABA}$ independent inhibition of PP2CA, HAI2, and HAI3. Moreover, the phenotype of PYL13 overexpression lines is similar to that of pp2ca-knockout mutants [34], whose seed germination is hypersensitive to ABA (Figure $4 \mathrm{E})$. According to previous reports, the germination of pp 2 ca- 1 mutant was about $45 \%$ under $0.5 \mu \mathrm{M} \mathrm{ABA}$ treatment and completely inhibited by $1 \mu \mathrm{M}$ ABA on day 5 after plating [34]. Our data showed that the germination of $O E-P Y L 13 \# 1$ was $56 \%$ under $0.5 \mu \mathrm{M}$ ABA treatment and $14 \%$ under $1 \mu \mathrm{M} \mathrm{ABA}$ treatment (Figure 4E). Consistently, the phenotype of pyll3 mutants is in the same pattern as PP2CA overexpression lines [34], whose seedling establishment is hyposensitive to ABA (Figure 4F). However, the phenotype of pyll3 mutants is very weak compared with $O E-P P 2 C A$ transgenic lines, indicating that there are functional redundancy between PYL13 and other PYLs in their inhibition of PP2CA. This is supported by the ABA-independent inhibition of PP2CA by transiently expressed PYL8 and PYL10 in protoplasts (Supplementary information, Figure S6) and by the recombinant PYL8 and PYL10 in phosphatase activity assays reported by other groups $[27,6]$. PYL8 and PYL10 inhibited approximately $30 \%$ and $50 \%$ of the phosphatase activity of PP2CA in the absence of ABA using a 10:1 ratio of PYLs:PP2CA [27]. Using a 4:1 ratio of
PYL8:PP2CA, a 10\%-15\% ABA-independent inhibition of PP2CA by PYL8 was reported [6]. These data demonstrated that PYL13 together with PYL8 and PYL10 regulate $\mathrm{PP} 2 \mathrm{CA}$ activity, which may explain the weak ABA phenotype of pyl13 single mutants. In OE-PYL13 seedlings, PYL13 may act as a constitutive activator of ABA signaling to maintain a higher basal level of ABA signaling under unstressed conditions and to enhance stress responses under stressed conditions, which would be advantageous in dealing with the unpredictable environmental changes. Indeed, we observed that transcripts of RD29A, KIN1, COR15A, and RAB18 were more abundant in $O E-P Y L 13$ seedlings than in the WT Col-0 under unstressed conditions and that the stress responses were enhanced in $O E-P Y L 13$ seedlings under ABA treatments (Figure $2 \mathrm{H}$ and $2 \mathrm{I}$ ). Thus, the improved drought resistance of PYL13 overexpression plants (Figure 2) may be explained by PYL13 inhibition of the PP2Cs in leaf tissues including the guard cells. The fact that we did not observe any significant defect in drought resistance in pyll3 mutant plants (data not shown) is consistent with the functional redundancy between PYL13 and other PYLs in regulating PP2CA.

In its second role, PYL13 antagonizes other ABAresponsive PYLs (Figure 6). PYL13 can interact with other PYLs independent of ABA in immunoprecipitation and mass spectrometric analyses (Supplementary information, Table S1), yeast two-hybrid assays (Figure 5A), LUC complementation (Figure 5B), and protein pull-down assays (Figure 5C). In the PYR/PYL/RCAR family, PYR1, PYL1 and PYL2 are homodimers, while PYLs 4-10 (except the untested PYL7) are monomers [27]. The monomeric mutant PYL2-I88K has a binding affinity with ABA about 7-fold higher than WT homodimeric PYL2 [27]. In the presence of ABA, dimeric PYLs need a conformational rearrangement to become monomers, which might be energy consuming. Heterodimer formation between PYL13 and other PYLs might reduce the binding affinity of other PYLs with ABA, thus antagonizing their activities. The heterodimer between PYL13 and other PYLs cannot be released by ABA, and this may interfere with the abilities of the other PYLs to bind to and inhibit PP2Cs. PYL13 can moderately reduce the activities of other PYLs in the presence of ABA as indicated by the analysis of ABI1 phosphatase activity (Figure 5D) and protoplast assays (Figure 5E). At later time points after ABA treatments, the stress-responsive genes maintain a similar expression level in OE-PYL13 transgenic plants and in WT plants (Figure 2H). PYL13 promoter::GUS reporter expression was detected in the roots, stems, leaves, flowers, imbibed seeds and guard cells (Supplementary information, Figure S7), indicating 
that PYL13 is highly expressed in these tissues. Although the expression level of PYL13 in different tissues relative to the other PYLs is not known at this time, these results indicate that PYL13 may rein in or desensitize ABA responses by antagonizing the ABA receptors. By acting as a "constitutive activator" and a moderate antagonist of other PYLs, PYL13 may help to maintain ABA signaling within a reasonable range.

Overexpression of $C B F 1$ induces $C O R$ genes and confers freezing tolerance [40]. Overexpression of $D R E$ $B 1 A / C B F 3$ induces DREB1, DREB2, and RD29A genes and confers increased resistance to drought, high-salt, and freezing stresses [41]. Although OE-OSMYB3R-2 exhibited enhanced cold tolerance in rice, it also resulted in growth retardation under normal conditions [42]. Similarly, OE-DREB $1 A$ in Arabidopsis caused severe growth retardation [41], and overexpression of $C B F 1$ in tomato resulted in a dwarf phenotype [43]. These results indicate that constitutive activation of stress-responsive genes increases stress tolerance but may also result in growth retardation. Thus, constitutive activation of stressresponsive genes may not be suitable for crop improvement. However, overexpression of PYL13 did not result in any abnormal growth phenotype under normal conditions. Under normal conditions, expression of the stressresponsive genes was only slightly higher in $O E-P Y L 13$ lines than in WT. Under stress conditions, however, these genes were induced more quickly in $O E-P Y L 13$ lines than in WT.

PYL13 differs from other PYLs in the highly conserved ABA-binding pocket CLs 1-4. Q38 differs from $\mathrm{K}$ in CL1, F71 differs from $\mathrm{L}$ in CL2, and T135 differs from N in CL4 (Supplementary information, Figure S1). We mutated Q38K, F71L, and T135N on PYL13 to mimic other PYLs. None of these single mutants could mimic PYR1 or PYL2 with respect to the activation of $R D 29 B$ $L U C$ expression in protoplasts. Double mutations of PYL13 (Q38K/F71L) and triple mutations (Q38K/F71L/ $\mathrm{T} 135 \mathrm{~N}$ ) could partially confer ABA-dependent induction of $R D 29 B-L U C$ expression (Figure 1B). These three residues of PYL13 are not conserved even in other dicots (Supplementary information, Figure S5), indicating that PYL13 may have evolved after the other PYLs. The residues Q38 and F71 in CLs 1-2 are critical for the unique mode of action of PYL13 and are conserved in the genus Arabidopsis (Supplementary information, Figure S5). T135 in CL4 can enhance the effect of Q38K/F71L in inhibiting ABI1 in the presence of ABA. These results suggest that the variations in the three residues $(\mathrm{Q} 38$ in CL1, F71 in CL2, and T135 in CL4) can partially explain the functional difference between PYL13 and the other PYLs, which are ABA receptors. According to the PYR1 structure, K59 in PYR1 interacts with ABA's carboxylic group [5], which is the corresponding position of Q38 in PYL13. This is consistent with our notion that Q38 is critical for ABA insensitivity of PYL13. In a screen for constitutively active receptors, PYR1 mutations located in 10 different residues were found to increase PYR1$\mathrm{HAB} 1$ interactions in the absence of ABA [38]. One of these mutations in PYR1 (L87F) is located at the corresponding position of F71L in PYL13. This is consistent with our notion that F71 is critical for ABA-independent binding between PYL13 and PP2Cs. Triple mutations of PYL13 (Q38K/F71L/T135N) abolished the ability of PYL13 to antagonize the activity of PYL5 in protoplasts (Figure $5 \mathrm{~F}$ ), indicating that the three amino acid variations are also important for the antagonistic role of PYL13. We also noticed that one special residue (F71) is in the corresponding position (L111) of PYL3, which is one of the five most important residues for trans-homodimer formation of PYL3 [44]. These results indicated that the heterodimer between PYL13 and other PYLs should be different from other reported PYL homodimers or trans-homodimers, although mechanistic insight into the dimerization or multimerization requires structural evidence.

Our results suggest that PYL13 is not an ABA receptor but it can inhibit both the PYL receptors and the PP2C co-receptors. The novel functions of PYL13 make it an important modulator of ABA signaling. Because overexpression of PYL13 increases drought resistance, photosynthetic rate, and water-use efficiency in transgenic plants, this naturally evolved ABA receptor variant could be useful for increasing the drought resistance of crops.

\section{Materials and Methods}

\section{Plant materials}

Arabidopsis thaliana Col-0 was used as WT. The pyl131 (SALK 041176) and pyl13-2 (SALK 071488) mutants were obtained from ABRC harboring T-DNA insertions in the exon of At4g18620. The insertion was confirmed by the gene-specific primers PYL13 CDSF and PYL13 CDSR, and the T-DNA (pROK2) left border-specific primers LBb1.3 (ATTTTGCCGATTTCGGAAC).

The PYL13 cDNA coding region was amplified by RT-PCR using the gene-specific primers PYL13 CDSF and PYL13 CDSR (Supplementary information, Table S2). The amplicon was cloned into the EcoRI and SalI sites of the binary vector pCAMBIA99-1 and confirmed by sequencing.

To generate the ProPYL13:PYL13-HA-YFP construct, the 2.79kb PYL13 promoter fragment amplified from Col-0 genomic DNA with the primers ProPYL13F and PYL13R (Supplementary information, Table S1) was cloned into the SalI and BamHI sites of the modified pSAT vector with YFP and 3HA tags at the C-terminus. The coding region of PYL13 from pCAMBIA99-1-PYL13 was 
then subcloned between the PYL13 promoter and the YFP coding sequence. The whole insertion cassette was digested with PI-Psp 1 and re-inserted into pRCS2-htp binary plasmids [45].

The resultant plasmids were transformed into Col-0 as mediated by Agrobacterium tumefaciens GV3101. All transgenic plants were verified by PCR and northern blot assays. The T3 generation was used for further experiments.

\section{Plasmid construction}

ZmUBQ::GUS was provided by J Sheen. RD29B::LUC, ABF2haemagglutinin (HA), SnRK2.6-Flag, and His-PYLs were the same as reported previously [19]. $A B F 2$ was replaced with $A B I 1, A B I 2$, HAB1, HAB2, AHG1, PP2CA, HAI1, HAI2, and HAI3 using transfer PCR [46] with pHBT-PP2Cs primers (Supplementary information, Table S2). PYR1 was replaced with PYL13 using transfer PCR with pHBT-PYL13 primers (Supplementary information, Table S2). The pHBT-His-PYL13 plasmid was used for mutagenesis with the QuikChange procedure (Stratagene) with specific primers (Supplementary information, Table S2). All plasmids were confirmed by sequencing.

PP2CA, ABI1, ABI2, AHG1, PYL13, and PYL2 were cloned into the pGEX-6P-1 vector between BamHI and EcoRI sites using transfer PCR with pGEX-PP2Cs or pGEX-PYLs primers (Supplementary information, Table S2).

\section{Transient expression assay in Arabidopsis}

Assays for transient expression in protoplasts were performed as described [4, 47]. Leaf strips $(0.5 \mathrm{~mm})$ were cut from the middle part of second leaves using a razor blade (VWR Scientific) and submerged in $15 \mathrm{ml}$ of enzyme solution containing $20 \mathrm{mM}$ MES, pH 5.7, 1.5\% (w/v) cellulase R10 (Yakult Pharmaceutical Industry), $0.4 \%(\mathrm{w} / \mathrm{v})$ macerozyme R10 (Yakult Pharmaceutical Industry), $0.4 \mathrm{M}$ mannitol, $20 \mathrm{mM} \mathrm{KCl}, 10 \mathrm{mM} \mathrm{CaCl}_{2}, 1 \mathrm{mM}$ $\beta$-mercaptoethanol, and $0.1 \%$ BSA. The leaves were vacuum infiltrated for $30 \mathrm{~min}$ and then incubated without shaking for $3 \mathrm{~h}$ in the dark at room temperature. The enzyme/protoplast solution was diluted with $15 \mathrm{ml}$ of W5 solution (2 mM MES, pH 5.7, $154 \mathrm{mM}$ $\mathrm{NaCl}, 125 \mathrm{mM} \mathrm{CaCl}_{2}$, and $5 \mathrm{mM} \mathrm{KCl}$ ) and filtered with a $75-\mu \mathrm{m}$ nylon mesh. Protoplasts were centrifuged at $100 \times g$ for $2 \mathrm{~min}$ in a 30-ml round-bottomed tube (Sarstedt), resuspended in W5 solution, and rested for $30 \mathrm{~min}$ at room temperature.

Before transfection, protoplasts were changed into MMG solution (4 mM MES, pH 5.7, 0.4 M mannitol, and $15 \mathrm{mM} \mathrm{MgCl}_{2}$ ) to a final concentration of $2 \times 10^{5}$ cells $/ \mu 1$. Protoplasts $(100 \mu \mathrm{l})$ were gently and thoroughly mixed with the plasmid DNA mixture (less than $10 \mu \mathrm{l})$ and $110 \mu \mathrm{l}$ of PEG solution (40\% w/v PEG-4000, 0.2 $\mathrm{M}$ mannitol, and $100 \mathrm{mM} \mathrm{CaCl}_{2}$ ), incubated for $5 \mathrm{~min}$, washed with $440 \mu \mathrm{l}$ of W5 solution, and resuspended in $50 \mu \mathrm{l}$ of WI solution (4 mM MES, pH 5.7, 0.5 M mannitol, and $20 \mathrm{mM} \mathrm{KCl).} \mathrm{After}$ transfection, protoplasts were incubated in WI solution without ABA or with $5 \mu \mathrm{M}$ ABA under light. The protoplasts were harvested after $4.5 \mathrm{~h}$, frozen in liquid $\mathrm{N}_{2}$, and stored at $-80^{\circ} \mathrm{C}$.

The frozen protoplasts were resuspended in $50 \mu \mathrm{l}$ of protoplast lysis buffer containing $2.5 \mathrm{mM}$ Tris-phosphate, $\mathrm{pH} 7.8,1 \mathrm{mM}$ DTT, 2 mM DACTAA, 10\% (v/v) glycerol, and 1\% (v/v) Triton $\mathrm{X}-100$. Protoplast lysates $(20 \mu \mathrm{l})$ were mixed with $100 \mu \mathrm{l}$ of LUC mix (Promega), and LUC luminescence was measured with a plate reader (Wallac VICTOR2 plate reader).

Protoplast lysates $(2 \mu \mathrm{l})$ were mixed with $10 \mu \mathrm{l}$ of 4-methylum- belliferyl $\beta$-D-glucuronide (MUG; Gold Biotechnology) substrate mix (10 mM Tris-HCl, $\mathrm{pH} 8,1 \mathrm{mM} \mathrm{MUG}$, and $2 \mathrm{mM} \mathrm{MgCl}_{2}$ ). After the mixture was kept for $30 \mathrm{~min}$ at $37^{\circ} \mathrm{C}, 100 \mu \mathrm{l}$ of $0.2 \mathrm{M}$ $\mathrm{Na}_{2} \mathrm{CO}_{3}$ was added, and GUS activity was measured using the plate reader with the excitation filter at $355 \mathrm{~nm}$ and the emission filter at $460 \mathrm{~nm}$.

All the plasmids used in this assay were purified with the QIAGEN Plasmid Maxi Kit or the QIAGEN Plasmid Midi Kit. The quality of plasmid DNA is critical for high transfection efficiency. $R D 29 B:: L U C$ (7 $\mu \mathrm{g}$ of plasmid per transfection) was used as the ABA-responsive reporter. $Z m U B Q: \because G U S$ ( $2 \mu \mathrm{g}$ of plasmid per transfection) was used as the internal control. $A B F 2-H A$, SnRK2.6Flag, PP2Cs-HA (except ABI1), and His-PYLs were used at $3 \mu \mathrm{g}$ per transfection. $A B I 1-H A$ was used at $2 \mu \mathrm{g}$ per transfection. HisPYL13 was used at $6 \mu \mathrm{g}$ per transfection when $2 \times$ PYL13 was needed.

\section{LUC complementation assay}

cLUC and nLUC were PCR amplified from pCAMBIA-NLuc and pCAMBIA-CLuc and cloned into pHBT95 to form pHBT95nLUC and pHBT95-cLUC using transfer PCR [46] with pHBTnLUC and pHBT95-cLUC primers (Supplementary information, Table S2). PYR1, PYLs 1-11, and PYL13 were amplified and cloned into pHBT-nLUC, and PYL13 was cloned into pHBTcLUC between BamHI and SalI using PYL primers (Supplementary information, Table S2). All plasmids were confirmed by sequencing.

All plasmids used in this assay were purified with the QIAGEN Plasmid Maxi Kit or the QIAGEN Plasmid Midi Kit. A 5- $\mu$ g quantity of purified plasmid was used per transfection. Protoplasts were incubated overnight in WI buffer containing 1\% LUC mix (Promega). The LUC luminescence of live protoplasts was measured in 96-well plates with a plate reader (Wallac VICTOR2 plate reader).

\section{Drought-stress treatment}

Seeds of the WT and OE-PYL13 lines were collected from plants subjected to the same growth conditions and periods. The seeds were imbibed at $4{ }^{\circ} \mathrm{C}$ for 4 days in the dark and planted directly in soil. Plants were grown in a growth room at $22 / 18{ }^{\circ} \mathrm{C}$ under a 14-h light/10-h dark photoperiod with a light intensity of 100 mmoles photons $\mathrm{m}^{-2} \mathrm{~s}^{-1}$. At 7 days after sowing (DAS), each pot was thinned to eight seedlings of uniform size. At 14 DAS, the drought-stress treatment was initiated by withholding water for 24 days, after which most WT plants had died. The plants were watered after 24 days of drought, and the surviving plants were counted 2 days later. The pots were rotated in the growth chamber every day to minimize the effect of environment. The complete experiment was repeated three times, each time with at least 3 replicates and at least 3 pots per replicate.

\section{Measurement of photosynthesis parameters and water loss}

Plants used for photosynthesis and water-loss assays were grown under short-day conditions (10-h light/14-h dark). After 5 days of drought stress treatment as described in the previous section, photosynthesis parameters were measured. Maximal rates of stomatal conductance, transpiration, and photosynthesis were measured with an infrared gas analyzer (6400, Li-Cor, Lincoln, 
NE, USA), and water-use efficiency was calculated. After photosynthetic measurements, leaf area was measured with a portable area meter $(3000 \mathrm{~A}, \mathrm{Li}-\mathrm{Cor})$. The leaves were then dried for $48 \mathrm{~h}$ at $65{ }^{\circ} \mathrm{C}$ and weighed. For water-loss determination, whole rosettes of 18-day-old plants were cut from the base and weighed at the indicated time points.

\section{Protein purification and pull-down assay}

Escherichia coli strain BL21 (DE3.0) was used to express the glutathione $S$-transferase (GST)-tagged recombinant proteins. Protein expression was induced by $1 \mathrm{mM}$ isopropyl $\beta$-Dthiogalactoside for $12 \mathrm{~h}$ at $22{ }^{\circ} \mathrm{C}$. GST-tagged proteins were purified from the soluble fraction using Glutathione Sepharose 4B (Amersham) under native conditions according to the manufacturer's instructions. The GST tag was removed by PreScission Protease at $4{ }^{\circ} \mathrm{C}$ overnight in PBS $(137 \mathrm{mM} \mathrm{NaCl}, 10 \mathrm{mM}$ phosphate, $2.7 \mathrm{mM} \mathrm{KCl}$, and $\mathrm{pH}$ 7.3) with $1 \mathrm{mM}$ DTT. His-tagged proteins were purified from the soluble fraction using PrepEase Histidinetagged High Specificity Purification Resin under native conditions according to the manufacturer's protocol.

In the His-mediated pull-down assay, His-PYL13 and HisPYR1 $(5 \mu \mathrm{g})$ were immobilized on PrepEase Histidine-tagged High Specificity Purification Resin (USB) and incubated with PP2Cs $(10 \mu \mathrm{g})$ with or without $200 \mu \mathrm{M}$ ABA at room temperature for $20 \mathrm{~min}$ in $1 \mathrm{ml}$ of binding buffer (PBS containing $0.1 \%(\mathrm{v} /$ v) Triton X-100). The agarose was rinsed four times with $1 \mathrm{ml}$ of binding buffer with or without $200 \mu \mathrm{M}$ ABA to remove unbound proteins, and the agarose was finally resuspended in $40 \mu \mathrm{l}$ of $1 \times$ SDS loading buffer (50 mM Tris-HCl, pH 6.8, 2\% SDS, $10 \%$ glycerol, $1 \% \beta$-mercaptoethanol, $12.5 \mathrm{mM}$ EDTA, $0.02 \%$ bromophenol blue) for $5 \mathrm{~min}$ at $100{ }^{\circ} \mathrm{C}$. A $10-\mu 1$ volume of each suspension was resolved by SDS-PAGE and Coomassie blue staining.

\section{Co-IP experiments in protoplasts}

$P P 2 C A-H A, A B I 1-H A$, and MYC-PYL13 plasmids (20 $\mu \mathrm{g}$ each) were transiently expressed in WT Col-0 protoplasts. After transfection, protoplasts were incubated in WI buffer under light at room temperature for $16.5 \mathrm{~h}$. Protoplasts were treated with $10 \mu \mathrm{M}$ ABA for $1.5 \mathrm{~h}$ before they were harvested, frozen in liquid $\mathrm{N}_{2}$, and stored at $-80{ }^{\circ} \mathrm{C}$. The frozen protoplasts were resuspended in $1 \mathrm{ml}$ of immunoprecipitation (IP) buffer containing $50 \mathrm{mM}$ Tris- $\mathrm{HCl}$, pH 7.4, $150 \mathrm{mM} \mathrm{NaCl}, 1 \mathrm{mM}$ EDTA, 0.1\% (v/v) Triton X-100, and $1 \times$ protease inhibitor cocktail (Sigma) with or without $10 \mu \mathrm{M}$ $\mathrm{ABA}$ and then centrifuged at $21000 \times \mathrm{g}$ for $30 \mathrm{~min}$ at $4{ }^{\circ} \mathrm{C}$. The supernatant was used as input and was incubated for $4 \mathrm{~h}$ at $4{ }^{\circ} \mathrm{C}$ with $30 \mu 1$ of monoclonal anti-HA-agarose antibody produced in mouse (Sigma, A2095). The agarose was washed four times using IP buffer with or without $10 \mu \mathrm{M}$ ABA and finally resuspended in $50 \mu \mathrm{l}$ of $1 \times$ SDS loading buffer for $5 \mathrm{~min}$ at $100{ }^{\circ} \mathrm{C}$. Protein samples $(8$ $\mu 1$ each) were resolved by SDS-PAGE and were electrotransferred onto PVDF membranes (Millipore). Membranes were blocked in blocking buffer (PBS containing 0.1\% Tween 20 (PBS-T) and 5\% skim milk) for $2 \mathrm{~h}$ at room temperature and incubated in blocking buffer containing 1:500 diluted anti-HA (Sigma, SAB4300603) or 1:2 000 diluted anti-c-MYC (Abcam, ab9106) rabbit antibodies for $1 \mathrm{~h}$ at room temperature. After they were washed five times (5 min each) with PBS-T, membranes were incubated for $1 \mathrm{~h}$ in blocking buffer containing 1:5000 diluted and stabilized goat antirabbit HRP-conjugated antibodies (Pierce, 32460). They were then washed five times with PBS-T and proteins were detected using Lumi-Light Western Blotting Substrate (Roche).

\section{Phosphatase activity assay}

Phosphatase activity was measured using the colorimetric substrate pNPP (Sigma). Reactions were performed in a reaction buffer containing $50 \mathrm{mM}$ Tris- $\mathrm{HCl}, \mathrm{pH} 7.5,25 \mathrm{mM} \mathrm{Mg}(\mathrm{OAc})_{2}, 2$ $\mathrm{mM} \mathrm{MnCl} 2,0.5 \mathrm{mM}$ EGTA, $0.5 \% \beta$-mercaptoethanol, and $0.5 \%$ BSA. ABA and PYLs were added as indicated. The concentration of PP2Cs and PYLs was $0.3 \mu \mathrm{M}$. Reactions were initiated by the addition of pNPP to a final concentration of $50 \mathrm{mM}$. The hydrolysis of pNPP was measured by the absorbance at $405 \mathrm{~nm}\left(\mathrm{~A}_{405}\right)$. Because His-PYL13 is difficult to purify, is prone to degradation, and rapidly loses activity, newly purified PYL13 was used for the phosphatase activity assay.

\section{Northern blot and real-time PCR}

For northern blot analysis, total RNA was isolated with the QIAGEN-RNeasy Mini Kit (Qiagen, Valencia, CA, USA) according to the manufacturer's instructions. Four microliters of RNA was loaded onto agarose gels containing formaldehyde, and the gels were run at 72 volts for $4 \mathrm{~h}$. After electrophoresis was completed, all RNA samples were transferred to Hybond- $\mathrm{N}^{+}$membrane by capillary transfer. Probes for the PYL13 gene (for verification of $O E-P Y L 13$ lines) and the YFP gene (for verification of ProPYL13-YFP-3HA transgenic lines) were labeled with the PCRDIG probe synthesis kit (Roche, Nutley, NJ, USA) according to the manufacturer's instructions. The following primers were used for PCR reactions: PYL13 forward: 5'-ATG GAA AGT TCT AAG CAA AAA C-3'; PYL13 reverse: 5'-TTA CTT CAT CAT TTT CTT TGT GAG-3'; YFP forward: 5'-ACG TAA ACG GCC ACA AGT TCA-3'; YFP reverse: 5'-ACT GGT AGC TCA GGT AGT GGT TGT-3'. Pre-hybridization, hybridization, washes, and detection were performed using the DIG high primer DNA labeling and detection starter kit II (Roche, Nutley, NJ, USA) according to the manufacturer's instructions.

Ten-day-old Arabidopsis Col-0 and OE-PYL13 seedlings were transferred to $1 / 2 \mathrm{MS}$ liquid medium, $\mathrm{pH}$ 5.8, with $100 \mu \mathrm{M}$ ABA. The seedlings were kept in the light and harvested at the indicated time points. For real-time PCR assays, reactions were set up with iQ SYBR Green Supermix (BioRad, CA, USA). The CFX96 Touch $^{\text {TM }}$ Real-Time PCR Detection System was used to detect amplification levels and was programmed for an initial step at $95{ }^{\circ} \mathrm{C}$ for $2 \mathrm{~min}$, followed by 40 cycles of $15 \mathrm{~s}$ at $95{ }^{\circ} \mathrm{C}$ and $1 \mathrm{~min}$ at 60 ${ }^{\circ} \mathrm{C}$. Quantification was performed with three independent experiments. Relative expression levels of target genes and SD values were calculated using the $2^{-\Delta \Delta C T}$ method [48]. Primers used for real-time PCR are provided in Supplementary information, Table S3.

\section{Promoter GUS analysis}

To generate PYL13 promoter::GUS construct, the PYL13 promoter (2 $395 \mathrm{bp}$ ) and coding region were amplified from Col0 genomic DNA with the following primers: GusF1 and GusR1, digested with SalI and NcoI; GusF2 and GusR2, digested with NcoI and BamHI, and cloned into the SalI and BamHI sites of the binary vector $\mathrm{pBI} 101$ and confirmed by sequencing. The resultant plasmid was transformed into Col-0 Arabidopsis mediated by Agrobacterium tumefaciens GV3101. 
For histochemical detection of GUS activity, whole seedlings or coatless embryos were immersed in freshly prepared reaction buffer containing $100 \mathrm{mM}$ sodium phosphate, $\mathrm{pH}$ 7.0, $10 \mathrm{mM}$ sodium EDTA, 1\% Triton X-100, $0.5 \mathrm{mM} \mathrm{K}_{3} \mathrm{Fe}(\mathrm{CN})_{6}, 0.5 \mathrm{mM} \mathrm{K}_{4} \mathrm{Fe}(\mathrm{CN})_{6}$ and $1 \mathrm{mM} 5$-bromo-4-chloro-3-indolyl- $\beta$-D-glucuronic acid (Xgluc; Sigma) and vaccum infiltrated briefly, and then incubated overnight in the dark at $37{ }^{\circ} \mathrm{C}$, and then washed with $70 \%$ ethanol several times until tissue is clear.

\section{Immunoprecipitation and mass spectrometry}

Proteins were extracted from Pro-PYL13-YFP-3HA transgenic lines and the Col WT with an extraction buffer containing $50 \mathrm{mM}$ Tris-HCl, pH 7.5, $150 \mathrm{mM} \mathrm{NaCl}, 5 \mathrm{mM}$ EDTA, 0.1\% NP-40, $10 \%$ glycerol, $1 \mathrm{mM}$ phenylmethylsulfonyl fluoride, $5 \mathrm{mM}$ DTT, and one unit of Protease Inhibitor Cocktail (Sigma-Aldrich, CA, USA). Immunoprecipitation was performed with GFP polyclonal antibody (Invitrogen, CA, USA). The protein complex was eluted from Protein A agarose beads with urea elution buffer (8 M Urea, $20 \mathrm{mM}$ Tris- $\mathrm{HCl}, \mathrm{pH} 7.5$, and $100 \mathrm{mM} \mathrm{NaCl}$ ). After in-gel digestion, the protein complex was identified using Q-TOF LC/MS/MS. MS/MS spectra were processed and queried against the NCBInr protein database using the MASCOT algorithm (http://www.matrixscience.com) for protein identification. Peptides were identified with a $\Delta$ mass value (observed mass - theoretical mass) less than $\pm 1.0 \mathrm{D}$

\section{Sequence comparison}

PYL13 homologs were obtained from The Arabidopsis Information Resource (TAIR, http://www.arabidopsis.org), DOE Joint Genome Institute (DOE JGI, http://www.jgi.doe.gov/), and Phytozome (http://www.phytozome.org/). Protein sequences were aligned using ClustalX 2.0.5 [49] with the default settings (Supplementary information, Data S1) and were viewed using GeneDoc software (http://www.nrbsc.org/gfx/genedoc/).

\section{Accession numbers}

Accession numbers of the proteins used in this study are provided in Supplementary information, Table S4.

\section{Acknowledgments}

This work was supported by US National Institutes of Health Grant R01GM059138 and by the Chinese Academy of Sciences.

\section{References}

1 Zhu JK. Salt and drought stress signal transduction in plants. Annu Rev Plant Biol 2002; 53:247-273.

2 Park SY, Fung P, Nishimura N, et al. Abscisic acid inhibits type $2 \mathrm{C}$ protein phosphatases via the PYR/PYL family of START proteins. Science 2009; 324:1068-1071.

3 Ma Y, Szostkiewicz I, Korte A, et al. Regulators of PP2C phosphatase activity function as abscisic acid sensors. Science 2009; 324:1064-1068.

4 Fujii H, Chinnusamy V, Rodrigues A, et al. In vitro reconstitution of an abscisic acid signalling pathway. Nature 2009; 462:660-664.

5 Santiago J, Dupeux F, Round A, et al. The abscisic acid re- ceptor PYR1 in complex with abscisic acid. Nature 2009; 462:665-668.

6 Antoni R, Gonzalez-Guzman M, Rodriguez L, Rodrigues A, Pizzio GA, Rodriguez PL. Selective inhibition of clade A phosphatases type $2 \mathrm{C}$ by PYR/PYL/RCAR abscisic acid receptors. Plant Physiol 2012; 158:970-980.

7 Gonzalez-Guzman M, Pizzio GA, Antoni R, et al. Arabidopsis PYR/PYL/RCAR receptors play a major role in quantitative regulation of stomatal aperture and transcriptional response to abscisic acid. Plant Cell 2012; 24:2483-2496.

8 Yin P, Fan H, Hao Q, et al. Structural insights into the mechanism of abscisic acid signaling by PYL proteins. Nat Struct Mol Biol 2009; 16:1230-1236.

9 Melcher K, Ng LM, Zhou XE, et al. A gate-latch-lock mechanism for hormone signalling by abscisic acid receptors. $\mathrm{Na}$ ture 2009; 462:602-608.

10 Miyazono K, Miyakawa T, Sawano Y, et al. Structural basis of abscisic acid signalling. Nature 2009; 462:609-614.

11 Melcher $\mathrm{K}, \mathrm{Xu} \mathrm{Y}, \mathrm{Ng} \mathrm{LM}$, et al. Identification and mechanism of ABA receptor antagonism. Nat Struct Mol Biol 2010; 17:1102-1108.

12 Soon FF, Ng LM, Zhou XE, et al. Molecular mimicry regulates ABA signaling by SnRK2 kinases and PP2C phosphatases. Science 2012; 335:85-88.

13 Brandt B, Brodsky DE, Xue S, et al. Reconstitution of abscisic acid activation of SLAC1 anion channel by CPK6 and OST1 kinases and branched ABI1 PP2C phosphatase action. Proc Natl Acad Sci USA 2012; 109:10593-10598.

14 Geiger D, Scherzer S, Mumm P, et al. Guard cell anion channel SLAC1 is regulated by CDPK protein kinases with distinct $\mathrm{Ca}^{2+}$ affinities. Proc Natl Acad Sci USA 2010; 107:80238028 .

15 Umezawa T, Sugiyama N, Mizoguchi M, et al. Type 2C protein phosphatases directly regulate abscisic acid-activated protein kinases in Arabidopsis. Proc Natl Acad Sci USA 2009; 106:17588-17593.

16 Vlad F, Rubio S, Rodrigues A, et al. Protein phosphatases 2C regulate the activation of the Snf1-related kinase OST1 by abscisic acid in Arabidopsis. Plant Cell 2009; 21:3170-3184.

17 Mustilli AC, Merlot S, Vavasseur A, Fenzi F, Giraudat J. Arabidopsis OST1 protein kinase mediates the regulation of stomatal aperture by abscisic acid and acts upstream of reactive oxygen species production. Plant Cell 2002; 14:3089-3099.

18 Fujii H, Verslues PE, Zhu JK. Identification of two protein kinases required for abscisic acid regulation of seed germination, root growth, and gene expression in Arabidopsis. Plant Cell 2007; 19:485-494.

19 Fujii H, Zhu JK. Arabidopsis mutant deficient in 3 abscisic acid-activated protein kinases reveals critical roles in growth, reproduction, and stress. Proc Natl Acad Sci USA 2009; 106:8380-8385.

20 Johnson RR, Wagner RL, Verhey SD, Walker-Simmons MK. The abscisic acid-responsive kinase PKABA1 interacts with a seed-specific abscisic acid response element-binding factor, TaABF, and phosphorylates TaABF peptide sequences. Plant Physiol 2002; 130:837-846.

21 Furihata T, Maruyama K, Fujita Y, et al. Abscisic acid-dependent multisite phosphorylation regulates the activity of a transcription activator AREB1. Proc Natl Acad Sci USA 2006; 
103:1988-1993.

22 Kwak JM, Mori IC, Pei ZM, et al. NADPH oxidase AtrbohD and AtrbohF genes function in ROS-dependent ABA signaling in Arabidopsis. EMBO J 2003; 22:2623-2633.

23 Sirichandra C, Gu D, Hu HC, et al. Phosphorylation of the Arabidopsis AtrbohF NADPH oxidase by OST1 protein kinase. FEBS Lett 2009; 583:2982-2986.

24 Vahisalu T, Puzorjova I, Brosche M, et al. Ozone-triggered rapid stomatal response involves the production of reactive oxygen species, and is controlled by SLAC1 and OST1. Plant $J$ 2010; 62:442-453.

25 Geiger D, Scherzer S, Mumm P, et al. Activity of guard cell anion channel SLAC1 is controlled by drought-stress signaling kinase-phosphatase pair. Proc Natl Acad Sci USA 2009; 106:21425-21430.

26 Lee SC, Lan W, Buchanan BB, Luan S. A protein kinasephosphatase pair interacts with an ion channel to regulate ABA signaling in plant guard cells. Proc Natl Acad Sci USA 2009; 106:21419-21424.

27 Hao Q, Yin P, Li W, et al. The molecular basis of ABA-independent inhibition of PP2Cs by a subclass of PYL proteins. Mol Cell 2011; 42:662-672.

28 Sun D, Wang H, Wu M, Zang J, Wu F, Tian C. Crystal structures of the Arabidopsis thaliana abscisic acid receptor PYL10 and its complex with abscisic acid. Biochem Biophys Res Commun 2012; 418:122-127.

29 Lackman P, Gonzalez-Guzman M, Tilleman S, et al. Jasmonate signaling involves the abscisic acid receptor PYL4 to regulate metabolic reprogramming in Arabidopsis and tobacco. Proc Natl Acad Sci USA 2011; 108:5891-5896.

30 Dupeux F, Antoni R, Betz K, et al. Modulation of abscisic acid signaling in vivo by an engineered receptor-insensitive protein phosphatase type 2C allele. Plant Physiol 2011; 156:106-116.

31 Nishimura N, Sarkeshik A, Nito K, et al. PYR/PYL/RCAR family members are major in-vivo ABI1 protein phosphatase 2C-interacting proteins in Arabidopsis. Plant J 2010; 61:290299.

32 Santiago J, Rodrigues A, Saez A, et al. Modulation of drought resistance by the abscisic acid receptor PYL5 through inhibition of clade A PP2Cs. Plant J 2009; 60:575-588.

33 Rubio S, Rodrigues A, Saez A, et al. Triple loss of function of protein phosphatases type $2 \mathrm{C}$ leads to partial constitutive response to endogenous abscisic acid. Plant Physiol 2009; 150:1345-1355.

34 Kuhn JM, Boisson-Dernier A, Dizon MB, Maktabi MH, Schroeder JI. The protein phosphatase AtPP2CA negatively regulates abscisic acid signal transduction in Arabidopsis, and effects of abh1 on AtPP2CA mRNA. Plant Physiol 2006; 140:127-139.

35 Chen H, Zou Y, Shang Y, et al. Firefly luciferase complementation imaging assay for protein-protein interactions in plants. Plant Physiol 2008; 146:368-376.
36 Peterson FC, Burgie ES, Park SY, et al. Structural basis for selective activation of ABA receptors. Nat Struct Mol Biol 2010; 17:1109-1113.

37 Dupeux F, Santiago J, Betz K, et al. A thermodynamic switch modulates abscisic acid receptor sensitivity. EMBO J 2011; 30:4171-4184.

38 Mosquna A, Peterson FC, Park SY, Lozano-Juste J, Volkman BF, Cutler SR. Potent and selective activation of abscisic acid receptors in vivo by mutational stabilization of their agonist-bound conformation. Proc Natl Acad Sci USA 2011, 108:20838-20843.

39 Bhaskara GB, Nguyen TT, Verslues P. Unique drought resistance functions of the highly ABA-induced Clade A protein phosphatase 2Cs. Plant Physiol 2012; 160:379-395.

40 Jaglo-Ottosen KR, Gilmour SJ, Zarka DG, Schabenberger O, Thomashow MF. Arabidopsis CBF1 overexpression induces COR genes and enhances freezing tolerance. Science 1998; 280:104-106.

41 Liu Q, Kasuga M, Sakuma Y, et al. Two transcription factors, DREB1 and DREB2, with an EREBP/AP2 DNA-binding domain separate two cellular signal transduction pathways in drought- and low-temperature-responsive gene expression, respectively, in Arabidopsis. Plant Cell 1998; 10:1391-1406.

42 Ma Q, Dai X, Xu Y, et al. Enhanced tolerance to chilling stress in OsMYB3R-2 transgenic rice is mediated by alteration in cell cycle and ectopic expression of stress genes. Plant Physiol 2009; 150:244-256.

43 Hsieh TH, Lee JT, Charng YY, Chan MT. Tomato plants ectopically expressing Arabidopsis cbf1 show enhanced resistance to water-deficit stress. Plant Physiology 2002; 130:618626.

44 Zhang X, Zhang Q, Xin Q, et al. Complex structures of the abscisic acid receptor PYL3/RCAR13 reveal a unique regulatory mechanism. Structure 2012; 20:780-790.

45 Tzfira T, Tian GW, Lacroix B, et al. pSAT vectors: a modular series of plasmids for autofluorescent protein tagging and expression of multiple genes in plants. Plant Mol Biol 2005; 57:503-516.

46 Erijman A, Dantes A, Bernheim R, Shifman JM, Peleg Y. Transfer-PCR (TPCR): a highway for DNA cloning and protein engineering. J Struct Biol 2011; 175:171-177.

47 Yoo SD, Cho YH, Sheen J. Arabidopsis mesophyll protoplasts: a versatile cell system for transient gene-expression analysis. Nat Protoc 2007; 2:1565-1572.

48 Livak KJ, Schmittgen TD. Analysis of relative gene expression data using real-time quantitative PCR and the 2(-Delta Delta C(T)) method. Methods 2001; 25:402-408.

49 Larkin MA, Blackshields G, Brown NP, et al. Clustal W and Clustal X version 2.0. Bioinformatics 2007; 23:2947-2948.

50 Hauser F, Waadt R, Schroeder JI. Evolution of abscisic acid synthesis and signaling mechanisms. Curr Biol 2011; 21:R346-R355.

(Supplementary information is linked to the online version of the paper on the Cell Research website.) 